\title{
Impact of Incorporation Kinetics on Device Fabrication with Atomic Precision
}

\author{
Jeffrey A. Ivie, ${ }^{1, \dagger}$ Quinn Campbellø,,${ }^{2, \dagger}$ Justin C. Koepke, ${ }^{1, \dagger}$ Mitchell I. Brickson, ${ }^{2,3}$ \\ Peter A. Schultz $\odot,{ }^{1}$ Richard P. Muller, ${ }^{1}$ Andrew M. Mounce, ${ }^{4}$ Daniel R. Ward $\odot, 1,+$ \\ Malcolm S. Carroll, ${ }^{1,}$ Ezra Bussmann $\odot,{ }^{1}$ Andrew D. Baczewski $\odot,{ }^{2,3, *}$ and Shashank Misra ${ }^{1}$ \\ ${ }^{1}$ Sandia National Laboratories, Albuquerque, New Mexico, USA \\ ${ }^{2}$ Center for Computing Research, Sandia National Laboratories, Albuquerque, New Mexico, USA \\ ${ }^{3}$ Center for Quantum Information and Control (CQuIC), University of New Mexico, Albuquerque, New Mexico, \\ USA \\ ${ }^{4}$ Center for Integrated Nanotechnologies (CINT), Albuquerque New Mexico, USA
}

(Received 22 July 2021; revised 3 August 2021; accepted 27 October 2021; published 18 November 2021)

\begin{abstract}
Scanning tunneling microscope lithography can be used to create nanoelectronic devices in which dopant atoms are precisely positioned in a Si lattice within approximately $1 \mathrm{~nm}$ of a target position. This exquisite precision is promising for realizing various quantum technologies. However, a potentially impactful form of disorder is due to incorporation kinetics, in which the number of $\mathrm{P}$ atoms that incorporate into a single lithographic window is manifestly uncertain. We present experimental results indicating that the likelihood of incorporating into an ideally written three-dimer single-donor window is $63 \pm 10 \%$ for room-temperature dosing, and corroborate these results with a model for the incorporation kinetics. Nevertheless, further analysis of this model suggests conditions that might raise the incorporation rate to near-deterministic levels. We simulate bias spectroscopy on a chain of comparable dimensions to the array in our yield study, indicating that such an experiment may help confirm the inferred incorporation rate.
\end{abstract}

DOI: 10.1103/PhysRevApplied.16.054037

\section{INTRODUCTION}

Atomic precision (AP) placement of individual dopant atoms in Si nanoelectronic devices is a promising avenue for realizing a variety of technologies ranging from analog quantum simulators [1-6], to qubits [7-13], to digital electronics $[14,15]$. This paper considers a particular limitation of AP donor placement with STM lithography [16-18] (see Fig. 1). STM lithography allows for the fabrication of devices in which single-donor atoms are positioned to within approximately $1 \mathrm{~nm}$ of a target lattice site [19]. This weak placement disorder has previously been predicted not to be of concern when it comes to the prospects for fabricating analog quantum simulators [5] or nuclear spin qubits [20] with this approach. However, these models do

\footnotetext{
*adbacze@sandia.gov

${ }^{\dagger}$ These authors contributed equally.

$\ddagger$ Present address: HRL Laboratories, LLC, Malibu, CA 90265, USA.

§Present address: IBM Quantum, IBM T.J. Watson Research Center, Yorktown Heights, NY 10598, USA.
}

Published by the American Physical Society under the terms of the Creative Commons Attribution 4.0 International license. Further distribution of this work must maintain attribution to the author(s) and the published article's title, journal citation, and DOI. not account for the failure of a precisely placed donor to incorporate. While STM lithography achieves AP placement of individual donor atoms, we show that a single precisely placed donor will incorporate with probability less than one for room-temperature dosing. While this is an indirect observation and quantitatively similar to previously reported results [21], our inference is corroborated by a kinetic model. That same model suggests conditions that might lead to deterministic incorporation.

Stochastic incorporation kinetics might have severe impacts for the fabrication of any device that relies on AP placement of a large number of single dopants. For certain applications, even perfect placement may be insufficient if a fixed fraction of the dopants simply are not there. Finding conditions that lead to deterministic incorporation is thus desirable. Analog quantum simulation of the Fermi-Hubbard model using donor arrays provides a useful example that both introduces the physics of interest and the potential impacts of stochastic incorporation. The physics that is most relevant to analog quantum simulation is the hydrogenic nature of shallow donors [22]. In its neutral ground state, a single bulklike $\mathrm{P}$ donor will have one weakly bound electron $45.59 \mathrm{meV}$ from the bulk conduction band edge [23-25]. The affiliated orbital will have an anisotropic effective Bohr radius that exceeds that of a hydrogen atom in vacuum [26]. This renormalization 
(a)

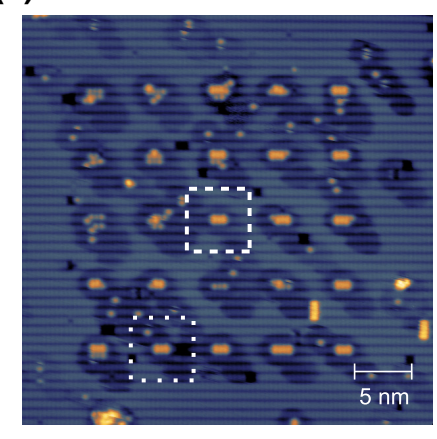

(b)

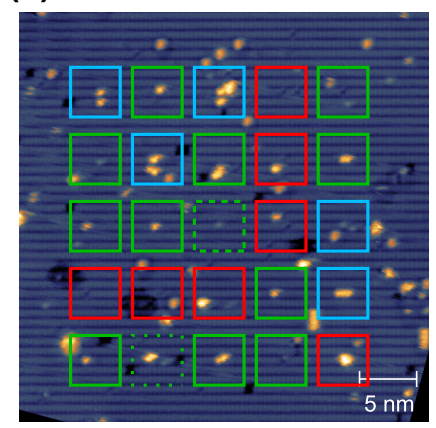

(c)

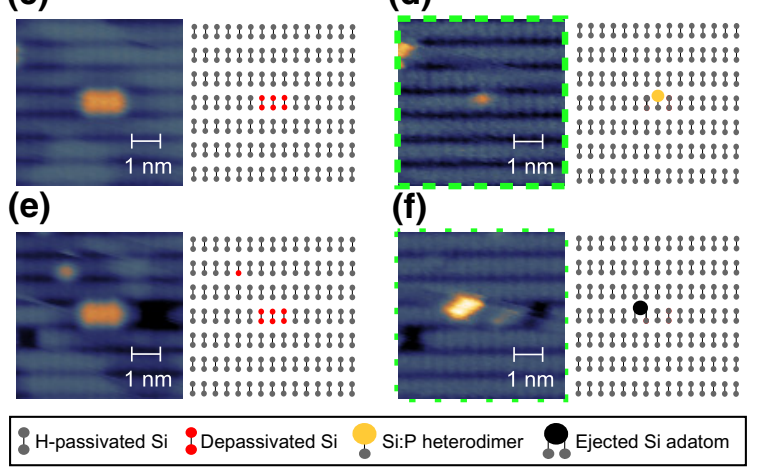

FIG. 1. Example STM lithography array. (a) STM image of 25 patterned windows, where the two marked windows correspond to (c),(e), respectively. (b) STM image of the same windows after $\mathrm{PH}_{3}$ dosing and incorporation. Each window is colored to indicate the number of $\mathrm{P}$ incorporation features in each window, with red corresponding to 0 , green corresponding to 1 , and blue corresponding to 2 . The corresponding windows after incorporation for (c),(e) are shown in (d) and (f), respectively. (c) Example $w=3$ window after hydrogen depassivation lithography corresponding to the middle window in (a). (d) Illustration of the window from (c) after incorporation where a Si:P heterodimer remains. It is likely that the corresponding Si adatom has diffused away. (e) Another example $w=3$ window after lithography, corresponding to the window in the lower left in (a). (f) Illustration of the window from (e) after incorporation where an ejected Si adatom is observed. This adatom feature is artificially broadened by a scan artifact and is likely masking any corresponding Si:P heterodimer feature.

is due to the bulk dielectric constant of the host crystal $\left(\epsilon_{\mathrm{Si}}=11.7\right)$ and the anisotropic conduction-band effective mass $\left(m_{\perp}=0.19 m_{e}\right.$ and $\left.m_{\|}=0.92 m_{e}\right)$. This artificial hydrogen atom can also be positively charged when it is stripped of its single weakly bound electron, or negatively charge when it is doubly occupied.

The low-energy effective theory that governs the behavior of the weakly bound electrons on a chain or array of such artificial hydrogen atoms is an extended FermiHubbard model $[4,5]$. The tunnel coupling between sites is determined by the physical distance between donor atoms and it is feasible to realize spacings that are within an order of magnitude of the effective Bohr radius. This can then realize instances of the extended Fermi-Hubbard model that are "hard" in the sense that there is competition between itinerance and localization of the weakly bound electrons.

Atomically precise arrays of more than approximately 50 donors are needed to realize a system that is large enough to represent an instance of the extended FermiHubbard model that exceeds the scale for which exact simulation on a classical computer is feasible [27]. Prior theoretical analyses $[4,5]$ have shown that the placement disorder realized in STM lithography is sufficiently weak to realize large arrays that exhibit the desired physics, assuming that each lithographic window yields exactly one $\mathrm{P}$ donor placed to within approximately $1 \mathrm{~nm}$. While current fabrication processes are evidently capable of reaching this precision in placement, the ultimate limits to their yield remains an open question.

To model the yield of STM lithography, we consider the probability of successfully fabricating an array of $N$ sites in which each site consists of $n=1$ donor,

$$
P(N)=\left(\sum_{w} P_{L}(w) P_{I}(n=1 \mid w)\right)^{N} .
$$

Here $P_{L}(w)$ is the probability of patterning a lithographic window consisting of $w$ dimers within a single row and $P_{I}(n \mid w)$ is the probability of incorporating $n$ donors into such a window. Implicit in this formula is the independence of success probabilities from site to site and the neglect of failure modes outside of lithography and incorporation chemistry [28]. Both assumptions make our formula an optimistic estimate. $P_{L}(w)$ is ultimately a function of the STM hardware and control software whereas $P_{I}(n \mid w)$ is a function of the surface chemistry of window $w$. To reliably fabricate arrays with $N>>1$ sites, i.e., $P(N) \approx 1$, it is evident that a window must be identified such that $P_{I}(n=1 \mid w)^{N} \approx 1$. The data in Fig. 2 suggest that even for the best choice of $w$, a window consisting of three dimers, $P_{I}(n=1 \mid w=3)=63 \pm 10 \%$. Even with perfect lithography and processing the probability of successfully fabricating an approximately 50-donor array for analog quantum simulation is approximately $10^{-10}$.

In what follows, we substantiate this dismal observation with a model of the incorporation chemistry. However, we also suggest a path forward. While it may at first seem as if only $P_{L}(w)$ can be driven arbitrarily close to 1 through improvements to the STM hardware and control software, our incorporation model indicates that dosing at lower pressures for longer times and/or slightly higher temperatures can lead to $P_{I}(n \mid w) \rightarrow 1$. Intuitively, the $w=3$ 
windows need sufficient time and space for a single $\mathrm{PH}_{3}$ molecule to land in the window without interference and then follow the correct pathway to a configuration in which incorporation occurs. Our model suggests that the stochastic incorporation that we observe is potentially a feature of room-temperature dosing. Because our incorporation statistics are based on inference, we briefly consider more direct measurable signatures of missing donors. We propose that bias spectroscopy measurements on a chain of dimensions comparable to the array fabricated in this work can yield insights into whether stochastic incorporation is occurring in a given process.

\section{RESULTS}

\section{A. STM experiments}

To generate a statistical sample sufficient to estimate $P_{I}(n \mid w)$ for different values of $n$ and $w, 50$ windows are patterned using standard STM lithography techniques (see Fig. 1 and Appendix A). Our aim is to pattern an array consisting entirely of $w=3$ dimer windows into which the incorporation of the $n=1 \mathrm{P}$ atom should be maximized. While feedback lithography would result in near-perfect patterning of any target window size [29-31], we do not make use of it. This would have slowed down patterning, potentially increasing susceptibility to atomic scale creep in the STM and limiting our ability to successfully pattern an array of the dimensions that are achieved. We also deem it useful to have estimates for $P_{L}(w)$ in an open-loop setting. Thus $P_{L}(w=3)$ is less than one but this is irrelevant for our analysis as subsequent scans of the depassivated windows are taken to establish $w$ for each window. After dosing and incorporation, STM scans of the areas containing the depassivated windows are taken again to determine $n$. Estimates for $P_{I}(n \mid w)$ are the relative frequencies of each observed $n$ for the measured $w$.
We note that STM scans of the predose and postincorporation surfaces are adjusted to bring the two images into registration. The postincorporation scan is aligned to the predose scan using intrinsic defects in the reconstructed $\mathrm{Si}(100)-2 \times 1$ surface as markers (see Appendix B). After alignment, each window in both the predose and postincorporation STM scans are extracted and gridded with respect to each Si lattice site (lattice constant $5.4 \AA$ ) for analysis. We expect that this alignment procedure results in a near-perfect overlap between the scans, where, in principle, only features present within a given window would be considered for evidence of incorporation. We allow for a single exception to this rule, where a $\mathrm{Si}$ :P heterodimer, as defined below, is allowed to be a single $2 \times 1$ dimer away from the window (Appendix B), as $\mathrm{Si}: \mathrm{P}$ heterodimers represent direct evidence of $\mathrm{P}$ incorporation. It should be noted that windows that demonstrated no change between STM scans of the postdosing and postincorporation surfaces are removed from the sample as incorporation cannot be established for such windows (total of 3).

The value of $w$ is determined for a given lithographic window by counting the number of contiguous depassivated $\mathrm{Si}$ atoms within a given dimer row. Depassivated atoms found in adjacent dimer rows or located noncontiguously to the primary pattern are excluded. If no more than one depassivated Si atom satisfied the criteria set above, then the pattern is considered disordered and removed from our sample. The variability in the number of depassivated $\mathrm{Si}$ atoms for the 47 windows is depicted in Fig. 2(a). Here and elsewhere we specify $w$, the number of depassivated dimers rather than atoms, resulting in half-integer values for dimers that are partially depassivated.

For our target $w=3$ pattern, only 20 windows are determined to meet the above criteria, i.e., $P_{L}(w=3)=$ $40 \%$. However, based on chemical considerations $[32,33]$ (a)

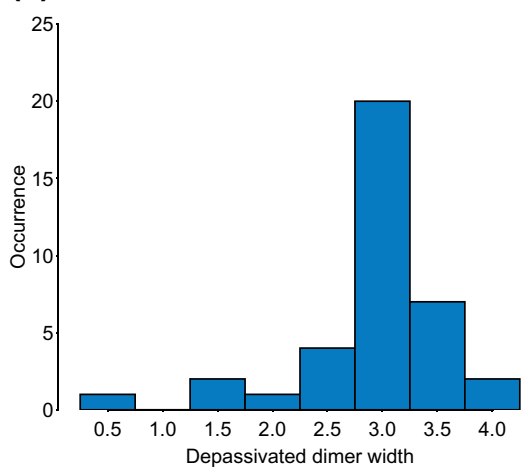

(b)

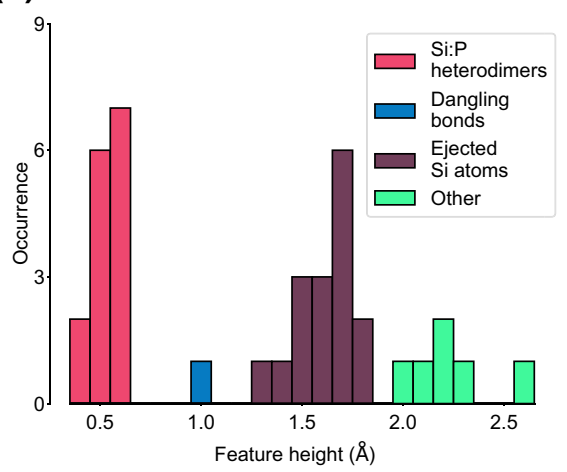

(c)

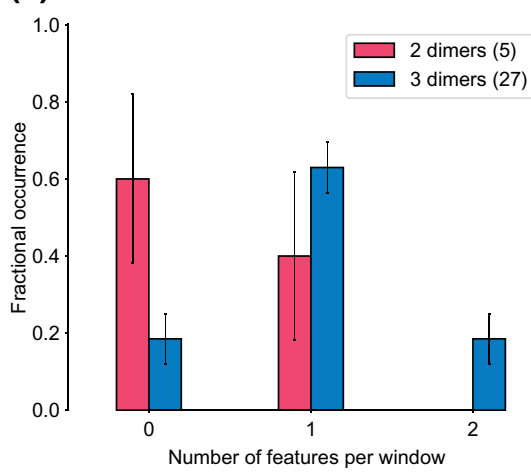

FIG. 2. Statistics for our 50-window sample. (a) The occurrence of different depassivation feature widths in the predose images for a target width of $w=3$. These data are used to assign $w$ for a particular window. (b) The occurrence of distinct feature categories according to their height. These data are used to assign $n$ for a particular window. (c) The relative frequencies, $P_{I}(n \mid w)$ for $n=0,1,2$ and $w=2,3$. The number of windows realizing $w=2,3$ are indicated parenthetically. 
the $w=3.5$ pattern should exhibit similar incorporation chemistry, and including these windows in our sample increases the number of windows that realize the target pattern to 27 , i.e., $P_{L}(w=3)=54 \%$. Thus the estimates of $P_{I}(n \mid w)$ for $w=2,3$ in Fig. 2 include the corresponding half dimer results (i.e., $w \rightarrow\lfloor w\rfloor$, see Appendix $\mathrm{C}$ for results separating the half-integer values of $w$ ).

To determine the number of donors in a given window from the postincorporation scans, it is necessary to categorize features according to their height relative to the surrounding $\mathrm{H}$ resist. The distribution of feature heights in the $w=2,3$ windows are shown in Fig. 2. For a single donor to have incorporated successfully, two features should be present $[33,34]$ - an incorporated $\mathrm{P}$ atom in the form of a $\mathrm{Si}: \mathrm{P}$ heterodimer and an ejected $\mathrm{Si}$ adatom. Based on methodology used previously in the literature [21], we assign the first feature height grouping to Si:P heterodimers, with a relative height of $0.3-0.6 \AA$ [34-36] and the second to $\mathrm{Si}$ adatoms, with a relative height of $1.2-1.8$ $\AA$ [21].

However, we also observe two feature categories that are not associated with successful incorporation. The first is a set of features with a relative height of $1 \AA$ that was previously assigned to depassivated $\mathrm{Si}$ atoms [37], consistent with height measurements of the predose surface. The second set of features have relative heights greater than $1.8 \AA$, which we ascribe to either background contamination in the UHV chamber or ejected Si adatoms adsorbed on $\mathrm{H}[38,39]$. We presume that these possible $\mathrm{Si}$ adatoms are purely adventitious as they never correspond to windows with zero features, and therefore we neglect them in further analysis.

Having assigned values of $w$ to each window and categorized the features in the postincorporation scans, we evaluate $P_{I}(n \mid w)$ from the relative frequencies of particular values of $n$. This requires us to assign values of $n$ to a particular window based upon the presence of particular features, specifically the Si:P heterodimer and $\mathrm{Si}$ adatom features. We note that both features are counted individually in Fig. 2(b). Due to the different diffusion barriers for the Si:P heterodimer [35,40] and the Si adatom [41,42], we do not expect that both features are necessarily present within a particular lithographic window. Thus, if either feature is found within a particular window it is counted toward an incorporation event. This brings us to one of our key results, that $P_{I}(n=1 \mid w=3)=63 \% \pm 10 \%$, consistent with previously reported statistics of $P_{I}(n=1 \mid w=$ $3)=70 \% \pm 8 \%$ for similarly sized windows [21]. Here we assign an uncertainty according to the standard error for a binomial distributed random variable with a success probability determined by the empirical frequency and a number of Bernoulli trials equal to the number of successfully patterned 3 and 3.5 dimer windows.

We note that we expect $P_{I}(n>1 \mid w=3)=0 \%$ due to a paucity of dangling bonds on which two $\mathrm{PH}_{3}$ molecules might shed four $\mathrm{H}$ atoms to arrive at a bridging $\mathrm{P}-\mathrm{H}$ configuration in which the $\mathrm{P}$ can incorporate. Two-donor incorporation requires at least $w=4$ depassivated dimers. However, we observe a small but nonzero probability for $P_{I}(n=2 \mid w=3)$. We hypothesize that this is a consequence of features diffusing into the window, rather than two donors incorporating within the same $w=3$ window. We suggest that a similar mechanism might also be biasing our estimate of $P_{I}(n=1 \mid w=2)$ upwards. We note that this bias is not accounted for in our quoted uncertainties.

Returning to Eq. (1), our results indicate that $P(N)=$ $(40 \% \pm 22 \%)^{N}$ for our particular process [43]. However, if $P_{L}(w=3) \rightarrow 100 \%$ using, e.g., feedback lithography, we see that $P(N) \rightarrow(63 \% \pm 10 \%)^{N}$. In both cases, the prospects for incorporating a large array for analog quantum simulation are dismal. To better understand these statistics we corroborate our results with an atomistic model that accounts for stochastic single-donor incorporation.

\section{B. Incorporation model}

We develop a kinetic Monte Carlo (KMC) model to estimate $P_{I}(n \mid w)$ and implement it in the KMCLib package [44]. Each KMC calculation is repeated 200 times with different seeds for the random number generator. The sample mean is reported for each result and error bars are calculated by assuming a binomial distribution of measured counts and using the standard error based on sample size. In all calculations, unless otherwise stated, we use the experimental dose and anneal temperatures, pressures, and times. Our model includes reaction barriers from Warschkow et al. [32] and Wilson et al. [33] with additional features described in Appendix D. Focusing particularly on the windows for which we expect single-donor incorporation, we compute $P_{I}(n \mid w)$ for $w=2,3$, and 4 . The predictions of our model are compared to our experimental results in Fig. 3(a). We match the ratio of half dimers included in each width bin to the measured ratio from this work.

For $n=1$ and $w=2,3$ our KMC model matches the experimental incorporation rate within error bars. In the three-dimer-wide window, single-donor case, in particular, we predict $P_{I}(n=1 \mid w=3)=59 \% \pm 2 \%$ compared to the measured value of $63 \% \pm 10 \%$. For each reaction within our model chemistry, the rate is governed by an attempt frequency and a reaction barrier according to the Arrhenius equation. As the rates are exponentially sensitive to the reaction barriers and errors approximately 0.1 $\mathrm{eV}$ are typical of the semilocal density functional theory (DFT) calculations that predicted them, we examine whether we can better reproduce experiment by comparably sized adjustments to our barrier heights. Rather than assessing the sensitivity of incorporation rates to 
(a)

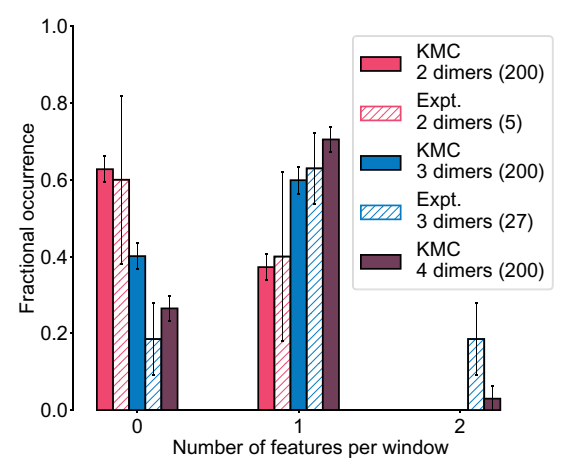

(b)

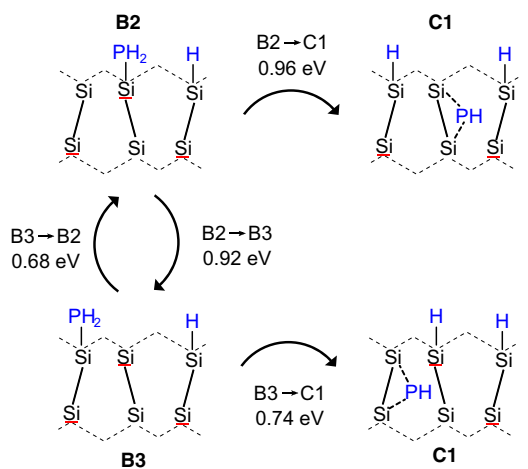

(c)

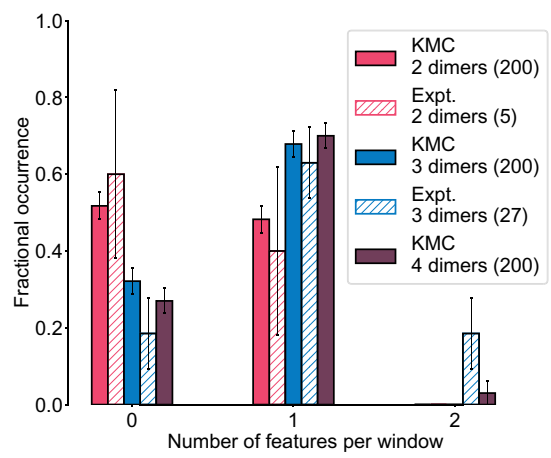

FIG. 3. Comparison of our incorporation model with our experimental results. (a) $P_{I}(n \mid w)$ calculated using KMC versus experimentally measured data. (b) P likely incorporates via two different pathways: a one-step process, or a two-step process with a thermodynamically unfavorable middle step. We indicate the lower end of the dimer row by underlining the Si label in red. Labels here refer to the notation used by Warschkow et al. [32]. (c) Incorporated P calculated using an instance of our KMC model in which the barrier of the $\mathrm{B} 3$ to $\mathrm{C} 1$ reaction is lowered by $0.1 \mathrm{eV}$. While there are minor changes in incorporation rates, our KMC model is largely robust to typical DFT errors in reaction barriers.

every barrier independently, we first identify particularly sensitive steps in the reaction pathway.

The two reaction pathways that dominate the incorporation of a $\mathrm{P}$ atom are depicted in Fig. 3(b). In the first pathway, outlined in Appendix $\mathrm{D}$, a $\mathrm{PH}_{2}$ molecule in a lower dimer end position (B2, using the naming conventions of Warschkow et al. [32]) moves into a bridging position between the two dimer atoms while simultaneously losing a hydrogen to the nearby dimer (C1). Alternatively, the $\mathrm{PH}_{2}$ molecule can first move to a nearby dimer, but on the raised end of the dimer (B3), before finally moving to a bridging $\mathrm{PH}$ position $(\mathrm{C} 1)$. This latter pathway involves a two-step process to incorporation, where the middle step is not thermodynamically favorable. Since the barrier for moving from $\mathrm{B} 2$ to $\mathrm{C} 1$ and the barrier for moving from $\mathrm{B} 2$ to $\mathrm{B} 3$ are within $0.05 \mathrm{eV}$ of each other, a system that starts in a $\mathrm{B} 2$ configuration has a roughly equal chance of moving directly to $\mathrm{C} 1$ and incorporating or moving to B3. From B3, the system again has a roughly equal chance of incorporating to $\mathrm{C} 1$ or transitioning back to B2.

This balance of options in the two-step pathway makes it critical to examine the impact of typical DFT error in calculating reaction barriers that increase the likelihood of moving from $\mathrm{B} 3$ to $\mathrm{C} 1$ instead of from $\mathrm{B} 3$ back to $\mathrm{B} 2$. In Fig. 3(c), we examine the impact of lowering the $\mathrm{B} 3$ to $\mathrm{C} 1$ barrier by $0.1 \mathrm{eV}$ while keeping all other reaction barriers constant. We find that lowering the barrier for the B3 to $\mathrm{C} 1$ reaction leads to $P_{I}(n=1 \mid w=3)=67 \% \pm 2 \%$. Further decreases to this reaction barrier eventually saturate before deterministic incorporation is achieved: lowering the $\mathrm{B} 3$ to $\mathrm{C} 1$ barrier by $0.2 \mathrm{eV}$, for instance, only results in $P_{I}(n=1 \mid w=3)=70 \% \pm 2 \%$. Our model is thus shown to be largely robust to typical errors in critical reaction barriers. For the remainder of this work, we revert to the original calculated reaction barriers in our model.

Accordingly, it is worth examining our model to see whether we can increase $P_{I}(n=1 \mid w=3)$ to $100 \%$. We also consider whether we can corroborate the number of donors in an incorporated device through measurements other than the number of ejected $\mathrm{Si}$ adatoms and $\mathrm{Si}: \mathrm{P}$ heterodimers.

\section{DISCUSSION}

\section{A. Potential for deterministic incorporation}

Dose time and pressure can also strongly affect incorporation and we present predictions using the kinetic model developed in the previous section for the incorporation rate as a function of both in Fig. 4. Notably the incorporation varies even at constant exposure, indicated as diagonal lines. A system at room temperature exposed to a 0.18 $\mathrm{L}$ dose of $\mathrm{PH}_{3}$ at a pressure of $3 \times 10^{-8}$ Torr for $6 \mathrm{~s}$ has $P_{I}(n=1 \mid w=3)=52 \% \pm 2 \%$, while the same exposure at a pressure of $3 \times 10^{-11}$ Torr for $6000 \mathrm{~s}$ has $P_{I}(n=$ $1 \mid w=3)=64 \% \pm 2 \%$. In general, moving toward conditions of lower pressure and longer dose time leads to increased incorporation.

In fact, for extreme conditions of low pressure and long time, we predict near-deterministic incorporation. At $3 \times$ $10^{-12}$ Torr for $6 \times 10^{5} \mathrm{~s}$ (approximately 6.9 days), $P_{I}(n=$ $1 \mid w=3)=99 \% \pm 1 \%$. Currently these conditions may be prohibitively difficult to achieve, thanks to the need for extreme ultrahigh vacuum and exceptionally pure $\mathrm{PH}_{3}$ dosing over several days, but it is instructive to understand the factors that lead our model to predict this.

Given enough space and time for a single $\mathrm{PH}_{3}$ molecule in a window to dissociate before another $\mathrm{PH}_{3}$ molecule 

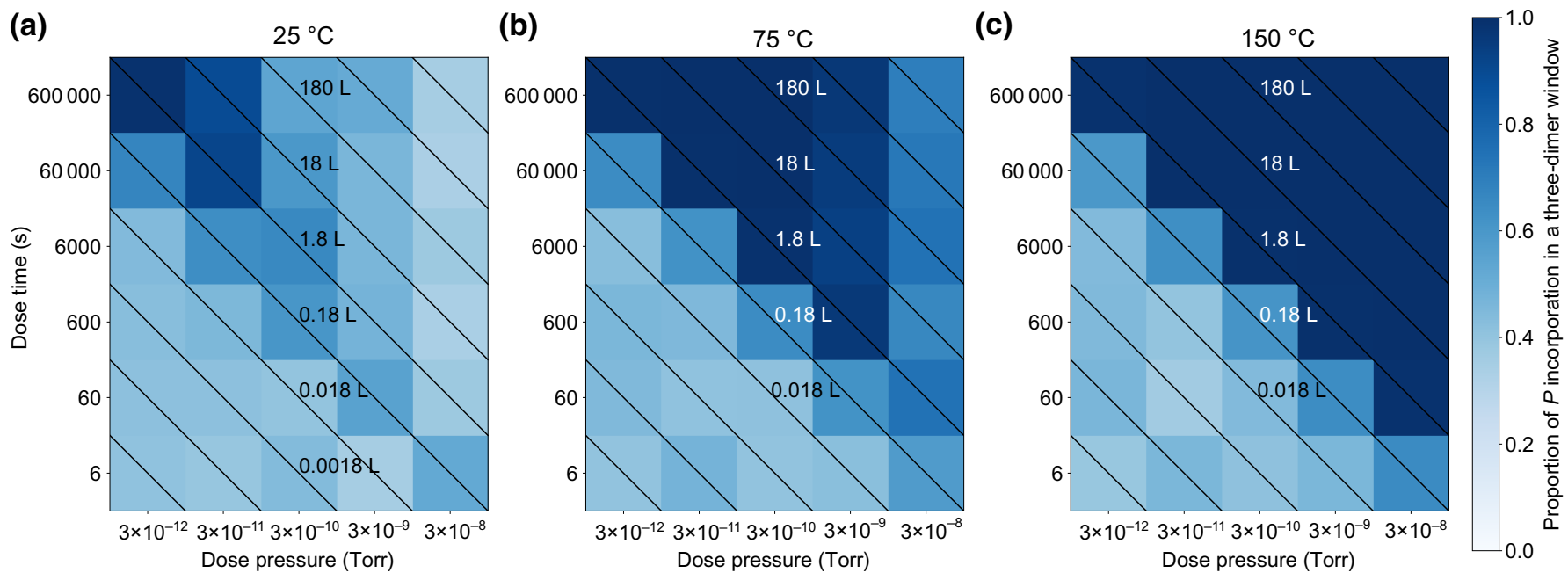

FIG. 4. The simulated probability of incorporation as a function of both dose time and pressure generated using the kinetic model outlined in Sec. B. Diagonal lines represent lines of constant exposure, indicated in langmuirs (L). Even within constant exposure, moving to low-pressure and long-time regimes can dramatically increase incorporation. Near-deterministic incorporation is predicted in the extreme low-pressure, long-time limit at room temperature. Incorporation can also be improved by raising the temperature during dosing as demonstrated by the heatmaps at (a) $25^{\circ} \mathrm{C}$, (b) $75^{\circ} \mathrm{C}$, and (c) $150{ }^{\circ} \mathrm{C}$.

adsorbs in the same space, incorporation will occur. The thermodynamic pathway for $\mathrm{PH}_{3}$ dissociation is entirely downhill. There are no dead ends in the path that would prevent it from eventually incorporating, only wrong turns that can be reversed given sufficient attempts. As pressure decreases, the likelihood of a single $\mathrm{PH}_{3}$ molecule adsorbing into a $w=3$ window fully dissociating before additional $\mathrm{PH}_{3}$ adsorption increases. At $3 \times 10^{-12}$ Torr, the average time for an incorporation event is $3.4 \times 10^{5} \mathrm{~s}$. If only one $\mathrm{PH}_{3}$ molecule adsorbs onto the $w=3$ window during the $6 \times 10^{5} \mathrm{~s}$ dose, that molecule will be able to shed its hydrogen and incorporate without any competition from neighboring molecules. In contrast, for a dose pressure of $3 \times 10^{-8}$ Torr, increasing the dose time can decrease the likelihood of incorporation due to additional phosphine molecules adsorbing into the window before the molecule has fully dissociated. Once $\mathrm{PH}_{3}$ evolves into a bridging $\mathrm{PH}$ configuration, however, the barriers for returning to a $\mathrm{PH}_{2}$ state are so high that any recombination is unlikely, even in the extreme time scales considered here. Moving from a $\mathrm{C} 1$ configuration to a $\mathrm{B} 3$ configuration requires overcoming a barrier of $1.86 \mathrm{eV}$, giving an average reaction time of $1.8 \times 10^{19} \mathrm{~s}$ at room temperature and $6.4 \times 10^{3} \mathrm{~s}$ at the anneal temperature of $320^{\circ} \mathrm{C}$.

Incorporation rates can therefore be increased by ensuring that the first $\mathrm{PH}_{3}$ molecule adsorbed on the surface dissociates before another $\mathrm{PH}_{3}$ molecule is adsorbed, blocking its pathway to incorporation. By increasing the sample temperature during dosing, the typical time for the dissociation reaction can be decreased significantly, as demonstrated in Figs. 4(b) and 4(c). At temperatures as low as $75^{\circ} \mathrm{C}$, near-deterministic incorporation is possible within a more typical range of pressures and temperatures, with conditions of $3 \times 10^{-10}$ Torr for $6000 \mathrm{~s}$ (approximately $1.6 \mathrm{~h}), P_{I}(n=1 \mid w=3)=98.8 \% \pm 1 \%$. This trend continues to expand at higher temperatures with all exposures $>1 \mathrm{~L}$ producing deterministic incorporation at $150^{\circ} \mathrm{C}$. Given the likelihood of hydrogen migration ruining the fidelity of the lithography window at higher temperatures, we expect that finding a feasible combination of pressure, time, and moderate temperature will produce the highest chance of success.

Our simulations predict that stochastic incorporation of a $\mathrm{P}$ donor in a $w=3$ window is not due to any inherent limitations of the $\mathrm{PH}_{3}$ chemistry. It is instead due to the limitations of current room-temperature dosing techniques. This suggests that heating the sample during dosing and/or the development of ultrapure chemical precursors and the ability to dose at pressures at or below $3 \times 10^{-12}$ Torr may make deterministic donor incorporation with atomic precision feasible using a $\mathrm{PH}_{3}$ chemistry. Our results also suggest that with current technology and dose temperatures, incorporation can still be increased by moving to lower pressures and longer dose times. We additionally consider the benefits of low-temperature (approximately $100 \mathrm{~K}$ ) dosing for deterministic incorporation in Appendix E.

\section{B. Corroborating incorporation statistics through transport measurements}

In assessing whether stochastic incorporation occurs in a given fabrication process, it is critical to consider other data that could support or refute incorporation statistics derived from observed Si adatoms and Si:P heterodimers. 
We already note that our inferred value of $P_{I}(n>1$ | $w=3)>0 \%$ is suspect and it is desirable to have a direct measurement of the electrically active substituted donors, more so because these are the technologically relevant elements. Bias spectroscopy is thus a natural choice and a powerful characterization technique that can be used to directly measure the spectrum of current-carrying manybody states in a donor array. By "bias spectroscopy" we mean the measurement of electron transport across the entire donor chain through the manipulation of conducting leads patterned in or out of plane of the chain, in contrast to local measurements of the donor chain using a scanning probe microscope. This measurement would have to be performed on a complete device, and could not be performed during fabrication. We consider the properties of a small donor array that might be probed with this technique to corroborate our incorporation statistics.

A bias spectroscopy measurement would require fabricating such a donor array between two $\delta$-doped nanowire source and drain leads with either another in-plane lead or top gate [45] to adjust the on-site potential across the array. To reduce the likelihood of confounding the measurement with sites consisting of $n>1$ donors, we propose making an array comprised exclusively of $w=3$ windows. Then it is likely the case that each site will consist of $n=0$ or $n=1$ donor. A one-dimensional chain with a short spacing between sites will be ideal. This spacing should be short enough that we can still expect conduction through the chain in case of missing sites. The number of sites in the chain should be both short enough to maintain good stability in the patterning and long enough to have a reasonable expectation of missing donors. A five-site chain strikes a balance between being long enough to have two missing sites (in expectation) and short enough to expect good tip stability in patterning. Indeed, a single row of the array in Fig. 1 would suffice. For such a chain, the spacing between sites would correspond to a nearest-neighbor tunnel coupling of approximately equal to $10 \mathrm{meV}$, diminishing to approximately equal to $1 \mathrm{meV}$ in the event of one missing site or approximately equal to $0.1 \mathrm{meV}$ for two consecutive missing sites [46]. Going to half this spacing is still viable and further increases the minimum tunnel coupling realized in the event of two missing sites by over an order of magnitude. AP patterning of the source and drain leads will allow us to precisely control the tunnel coupling onto or out of the chain. This distance should be determined to realize a tunnel coupling onto or out of the chain that is comparable to or weaker than the nearest-neighbor tunnel coupling on the chain. This limit is critical to the bias spectra being representative of the many-body states of the chain and will be robust to asymmetry or weak placement errors in the edge of either lead. We consider simulations of such a chain in Fig. 5.

Our simulated bias spectra are intended to be qualitatively illustrative of the impacts of missing sites.

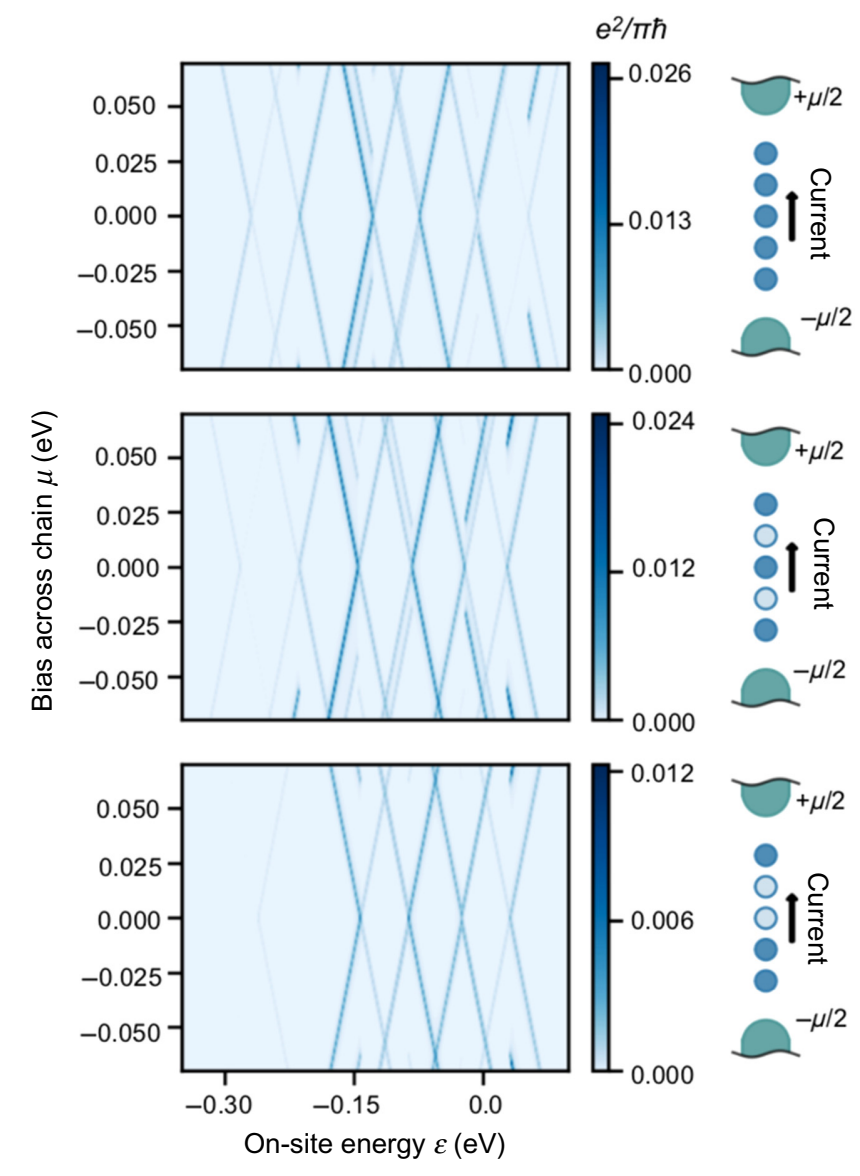

FIG. 5. Plots of the differential conductance (DC), $d I / d \mu$, as a function of the source-drain bias across the chain, $\mu$, and a uniform shift in the orbital energies, $\epsilon$, caused by a nearby gate voltage. The geometry of exemplary devices are illustrated on the right side of each plot, with the source (top) and drain (bottom) biased as to pass a current across a chain of single incorporated donors (dark) including patterned sites in which incorporation failed (light). (a) DC across an ideal chain of five sites with five donors. (b) DC across a chain of five sites in which every other donor is missing. (c) DC across a chain of five sites in which two consecutive donors are missing. In all cases, the faint transitions at higher occupancies (i.e., on the left of the plots) are diminished due to the increased impact of the intersite Coulomb interaction. We note that our calculations do not include current through scattering states to clearly demarcate the transport through the eigenstates of the chain and thus our Coulomb diamonds do not have signatures of transport "above" or "below" them, as would be expected in a real experiment.

We present an exhaustive enumeration of all possible missing donors, as well as other spacings and orientations that may be of experimental interest in Appendix F. Here we simply compare the spectrum of the ideal chain to the most likely configurations consistent with $P_{I}(n=1 \mid w=$ 3 ) $=63 \pm 10 \%$ (i.e., two missing donors). We expect the spectrum for the chain in which every other donor is missing (one of the ten possible configurations for a five-donor 
chain) to be qualitatively similar to that of the unbroken chain in this regime, with four prominent diamonds. But the majority of configurations' bias spectra (nine of ten) will more closely resemble the case in which there are two consecutive donors and two missing sites, with three prominent diamonds, as demonstrated in Appendix F. We thus expect that even in conditions favorable to tunnel coupling through missing donor sites, missing donors consistent with an incorporation rate of approximately $60 \%$ can be clearly detected with bias spectroscopy in $90 \%$ of possible configurations for a five-donor chain. A larger spacing will yield even greater sensitivity to the precise locations of the missing donors.

A quantitatively accurate prediction of the current through a few-donor chain would require some improvements to our model that are discussed in Appendix F. However, we expect the basic principle illustrated in Fig. 5 to hold - for a sufficiently short chain there will still be measurable Coulomb diamonds even in the presence of missing donors and deviations from the transport signatures expected in the ideal chain might herald a particular pattern of missing donors. We note that the transport signatures of missing donors are stronger for calculations using the traditional Fermi-Hubbard model (i.e., without the intersite Coulomb interaction). In that limit, the incipient Hubbard bands above and below half filling give indications that clearly reflect the longest unbroken chain. Because the intersite Coulomb interaction in the extended FermiHubbard model suppresses the formation of these incipient bands in small chains and breaks the symmetry between the bands above and below half filling, we expect these signatures to be a bit messier. Accordingly, we also expect that transport measurements of a chain of this dimension would provide experimental confirmation that the intersite term is a critical feature to include in effective models of Si:P arrays. We ultimately aim to use simulated bias spectra to infer plausible geometries for incorporated donor chains, though we leave the development of such a highfidelity simulation capability as a topic for future work.

\section{CONCLUSION}

We demonstrate that even for perfectly patterned lithographic windows, single-donor incorporation is stochastic with a $63 \pm 10 \%$ likelihood of success at roomtemperature dosing conditions, which we corroborate with a kinetic model. We thus conclude that building classically intractable single-donor arrays is overwhelmingly unlikely under these conditions. Our model nevertheless suggests that near-deterministic incorporation may be possible above room temperature or at low-pressure, long-time dosing conditions. We also consider bias spectroscopy as a method to directly measure the number of incorporated donors in an array, demonstrating that irregularities in the pattern of Coulomb diamonds can give clear indications of missing donors.

It is also worth remarking on the prospects of using AP technologies to realize analog quantum simulators with single acceptors [47,48], in addition to their prospective use in qubit technologies $[49,50]$. The Fermi-Hubbard model parameters for a pair of substitutional B dopants have been measured in a sample prepared without AP placement [51]. With the recent demonstration of $\mathrm{B}_{2} \mathrm{H}_{6}$ as a precursor for delta doping [52], it is clear that AP placement of individual $\mathrm{B}$ atoms might also be possible. An incorporation model for $\mathrm{B}_{2} \mathrm{H}_{6}$, similar to the one in this paper, suggests that the tendency for dimerized $\mathrm{B}$ to incorporate as electrically inactive might limit the prospects for achieving deterministic single B-atom placement for analog quantum simulation [53]. This is qualitatively different from the case presented here for $\mathrm{PH}_{3}$, in which we predict that changing the pressure and temperature of the $\mathrm{PH}_{3}$ dosing conditions can ultimately lead to deterministic AP placement. Nevertheless, modeling predicts that other precursors [54] and halogen resists might provide a path forward [53] and recent work is beginning to explore the vast space of options for AP fabrication [55-58].

Finally, it is worth emphasizing that stochastic incorporation is not necessarily a no go for the application of STM lithography to realizing analog quantum simulators, qubits, or digital electronics. A simple workaround is to target the fabrication of multidonor clusters [7,59] to realize quantum dots with precise dimensions. While our results suggest that there will be uncertainty in the number of donors in such a cluster, it may be that there is a "sweet spot" in target cluster dimensions for which the resulting variance in the resulting application-relevant properties (e.g., tunnel couplings, charging energies, etc.) is sufficiently low. In this context, the single-donor limit is somewhat pathological as there is a significant probability of not fabricating a cluster at all. The demonstration of a process that reliably realizes deterministic incorporation of a single donor into a three-dimer window, such as suggested by our modeling, would obviate some of the need for these considerations.

\section{ACKNOWLEDGMENTS}

We gratefully acknowledge useful conversations with Evan Anderson, Josh Ballard, Ed Bielejec, DeAnna Campbell, Udi Fuchs, John Gamble, Toby Jacobson, Ehsan Khatami, Dwight Luhman, Michael Marshall, Leon Maurer, Andrea Morello, James Owen, John Randall, Steve Rinaldi, Scott Schmucker, Joe Simonson, Lisa Tracy, Rick Silver, and Esmeralda Yitamben. We are also grateful to the two anonymous referees whose comments are incorporated into this paper. This work is partially supported by the Laboratory Directed Research and Development program at Sandia National Laboratories under projects 
209242 (DQM), 213017 (FAIR DEAL), and 213048 (Quantum ACCESS). This work is also performed, in part, at the Center for Integrated Nanotechnologies, a U.S. DOE, Office of Basic Energy Sciences user facility. Sandia National Laboratories is a multimission laboratory managed and operated by National Technology and Engineering Solutions of Sandia, LLC, a wholly owned subsidiary of Honeywell International, Inc., for DOE's National Nuclear Security Administration under Contract No. DE-NA0003525.

J.A.I., Q.C., and J.C.K. contributed equally to this work. E.B., S.M., and M.S.C. coordinated the experimental effort. A.D.B. coordinated the theoretical effort and the writing of the paper. J.C.K. planned and conducted the experiments generating the STM data. D.R.W. made critical advances to fabrication. J.A.I., J.C.K., Q.C., and A.M.M. analyzed the experimental data. Q.C., P.A.S., and R.P.M. developed the model for donor incorporation. M.I.B. carried out the transport calculations.

\section{APPENDIX A: DETAILS OF STM EXPERIMENTS}

Experiments are performed in an Omicron ultrahigh vacuum (UHV) variable-temperature STM (VT-STM) system with a XA microscope stage (base pressure < $5 \times 10^{-10}$ Torr), plumbed directly with phosphine $\left(\mathrm{PH}_{3}\right.$, 99.9\%) and hydrogen $\left(\mathrm{H}_{2}, 99.999 \%\right)$ gas lines. $\mathrm{Si}(100)$ samples (miscut $\leq 0.1^{\circ}$ ), etched with appropriate alignment markers for pattern realignment, are prepared ex situ by a wet chemical clean involving chemical oxidation (3:1 $\left.\mathrm{H}_{2} \mathrm{SO}_{4}: \mathrm{H}_{2} \mathrm{O}_{2} 90^{\circ} \mathrm{C}, 5 \mathrm{~min}\right)$, and reduction $\left(10: 1 \mathrm{H}_{2} \mathrm{O}: \mathrm{HF}\right.$, $10 \mathrm{~s}$ ), with rinsing with deionized $\mathrm{H}_{2} \mathrm{O}$ after each step, followed by successive sonications in methanol, acetone, and isopropyl alcohol for $10 \mathrm{~min}$. After wet chemical cleaning, samples are dried with dry $\mathrm{N}_{2}$ and immediately loaded into the UHV chamber. Samples are degassed at $625^{\circ} \mathrm{C}$ for $24 \mathrm{~h}$, then annealed for $10 \mathrm{~s}$ at $1100^{\circ} \mathrm{C}$ to achieve a clean $\mathrm{Si}(100) 2 \times 1$ reconstructed surface. Temperatures are recorded with an IR pyrometer (Metis MP-25), with $50^{\circ} \mathrm{C}$ accuracy for temperatures $>600^{\circ} \mathrm{C}$.

Samples are terminated with a monohydride resist for STM lithography by heating the sample to $350^{\circ} \mathrm{C}$ while exposing to atomic $\mathrm{H}$ supplied by cracking $\mathrm{H}_{2}\left(2 \times 10^{-6}\right.$ Torr, $10 \mathrm{~min}$ ) with a $\mathrm{W}$ filament positioned $1 \mathrm{~cm}$ away from the sample. All STM operations utilized PtIr STM tips from NaugaNeedles (NN-USPtIr-W250, radius of curvature $25-50 \mathrm{~nm}$ ), degassed at approximately $175^{\circ} \mathrm{C}$ for $30 \mathrm{~min}$ and further heated by electron bombardment using a commercial Omicron tip-preparation tool. All AP patterns are generated using $+3.5-\mathrm{V}$ bias, $6.0 \mathrm{nA}$ tunneling current, $6.0 \mathrm{mC} / \mathrm{cm}$ dose, and $10 \mathrm{~nm} / \mathrm{sec}$ tip speed using a 20-bit ZyVector Control System (Zyvex Labs). Before lithography, the underlying atomic lattice is identified and registered in the SCANZ control software, enabling AP patterning of individual dimer sites. Further details of comparable lithographic procedures can be found in Refs. $[16,17,60]$.

After lithography, dosing is performed with the sample in the STM UHV chamber. $\mathrm{PH}_{3}$ is introduced into the STM UHV chamber via a precision leak valve, with $\mathrm{PH}_{3}$ coverage calculated using the partial pressure of the $\mathrm{PH}_{3}$ introduced during dosing measured by a residual gas analyzer (RGA), specifically the $34 \mathrm{amu}$ fragment). In a typical process, a $\mathrm{PH}_{3}$ partial pressure of $3.0 \times 10^{-10}$ Torr is introduced for $10 \mathrm{~min}$ at room temperature, resulting in a coverage of $0.15 \mathrm{~L}$, which has been previously demonstrated to provide sufficient coverage for atomically precise donor structures $[61,62]$. While the partial pressure reported through the $\mathrm{PH}_{3} \mathrm{RGA}$ value may represent a lower bound on the actual partial pressure introduced, the reported dosing conditions have also been demonstrated to provide the expected $0.25 \mathrm{ML}$ coverage on a clean $\mathrm{Si}(100)$ surface for $\mathrm{P}$ incorporated at $310^{\circ} \mathrm{C}$ [63], further demonstrating that the dosing conditions utilized will result in sufficient coverage in the depassivated windows. A subsequent $310^{\circ} \mathrm{C}$ anneal is used to incorporate $\mathrm{P}$ into $\mathrm{Si}$ lattice sites, demonstrated to be sufficient for full donor incorporation [64].

All STM scans shown are taken under $-2.75 \mathrm{~V}, 300 \mathrm{pA}$ imaging conditions.

\section{APPENDIX B: ANALYSIS OF STM IMAGES}

In order to determine $P_{I}(n \mid w)$, the predosing and postincorporation datasets had to be "atomically registered" to each other such that each lattice site corresponds to the same pixel(s) in both datasets. This is accomplished by analyzing and transforming data sets with OpenCV followed by labeling with a custom labeling graphical user interface as detailed in the following.

The first step is to ensure that the predosing to postincorporation data sets had the same pixel-to-atomic site scaling. This is accomplished by finding the lattice periodicity via Fourier transform and scaling the postincorporation data to match the scaling of the predosing data sets. We then use the lattice defects as alignment markers between the two data sets by thresholding both data sets and nulling out all data above the approximate height of the defects. A correlation function is applied to the datasets to find the best overlap. Occasionally this produced an insufficient matching and an affine transform is produced by matching three specific defects across the images. Both methods produced a transform function that effectively "atomically registered" the predosing and postincorporation datasets to one another.

After the images had been "atomically registered," the datasets are segmented into small regions around the depassivation window and corresponding postincorporation area. These subimages are loaded into a custom labeling graphical user interface where the authors shifted 
(a)

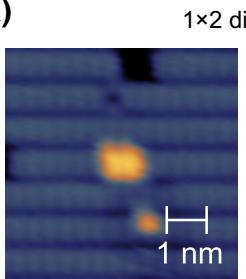

(b)

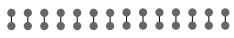

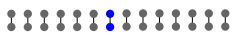

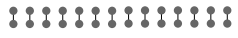

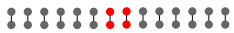

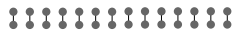

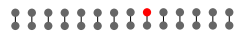

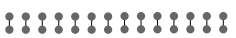

(d)

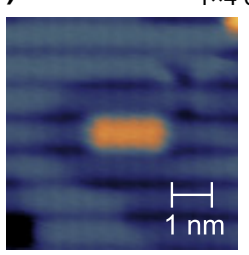

$\times 4$ dimers

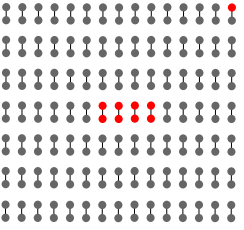

$1 \times 2.5$ dimers

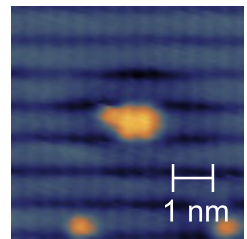

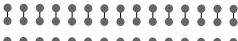

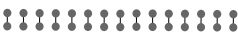

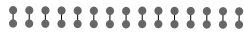

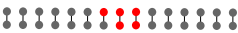

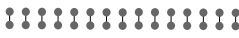

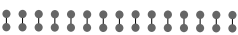

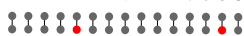

(c)

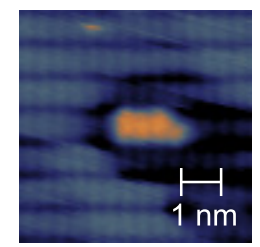

$1 \times 3.5$ dimers

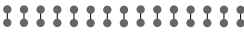

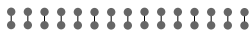

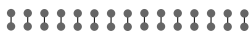

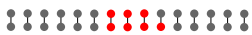

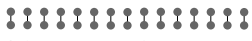

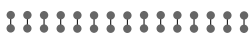

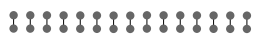

(e)

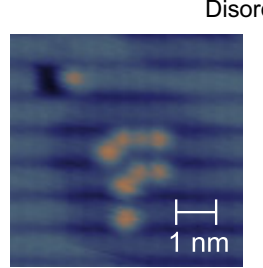

$:: !:: !: !: !: !: !: !$

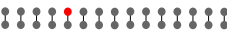

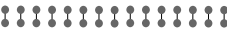

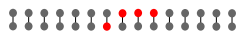

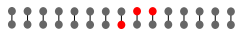

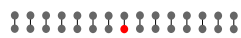
$::: 1: !: !: !: !: !:$

\section{\&H-passivated Si \&Depassivated Si \&Dihydride}

FIG. 6. Common lithographic outcomes.

each subimage to adjust the image so that each lattice site corresponded to an $8 \times 8$ set of pixels. Each lattice site is labeled as $X X X, Y Y Y$ through analysis of the contrast relatively to the neighboring lattice sites in the STM topographical image. In order to account for uncertainty in both the registration and feature height assignment procedures, both $\mathrm{Si}$ ad atom and $\mathrm{Si}: \mathrm{P}$ heterodimer features within a lithographic window are labeled as well as Si:P heterodimer features found within a single $2 \times 1$ dimer away from a given window. Such a $\mathrm{Si}: \mathrm{P}$ heterodimer is included in the analysis only if neither a $\mathrm{Si}$ ad atom or $\mathrm{Si}: \mathrm{P}$ heterodimer is found within the lithographic window, and is considered conclusive evidence of $\mathrm{P}$ incorporation as $\mathrm{Si}: \mathrm{P}$ heterodimers can result only from $\mathrm{P}$ incorporation. Several features, which would correspond to $\mathrm{Si}$ ad atoms, are rarely found to be located close to a patterned windows (within several $2 \times 1$ dimers) where no features are assigned, however, as no $\mathrm{Si}: \mathrm{P}$ heterodimers are found within those respective windows or surrounding them, we do not conclude that these ad atoms indicate the presence of $\mathrm{P}$ incorporation. After labeling, each labeled site is located within the overall postincorporation scan, with the corresponding feature height measured relative to the surrounding interdimer $\mathrm{H}$ resist. In both the predosing and postincorporation scans, care is taken to avoid incorrect height assignments resulting from "halo" that surrounds dangling bonds and other absorbates on the H-terminated (a)
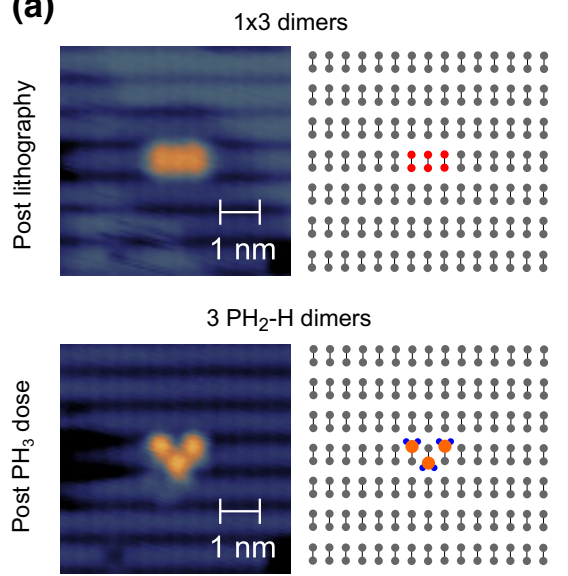

dimers

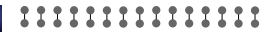

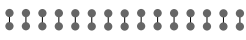
$: \div: !: !: !: !: !: !:$

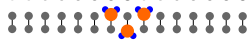

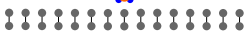

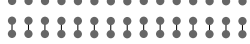

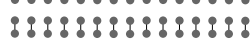

(b)
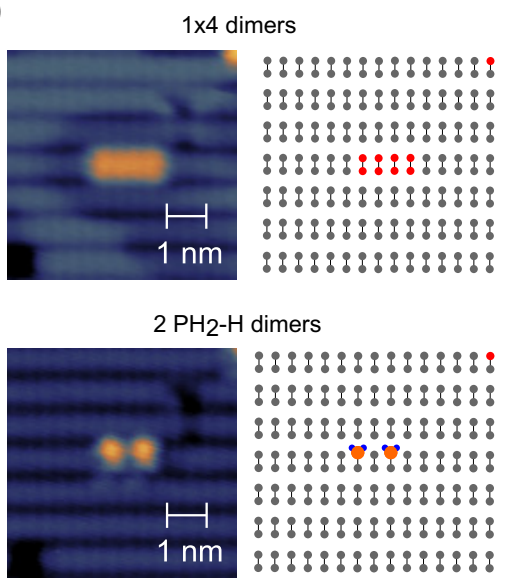

(c)

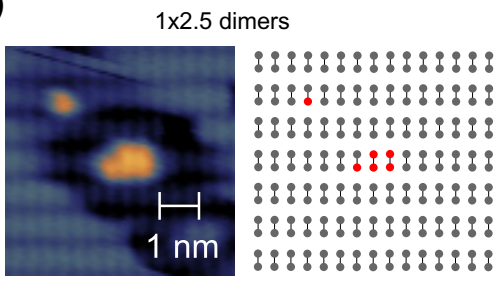

Repassivated

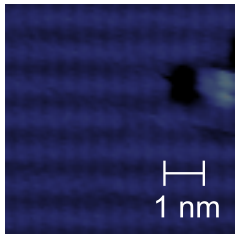

$: \because 2: 19: 19: 19: 18$

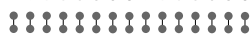

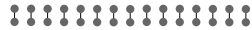

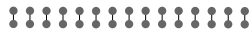

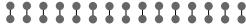

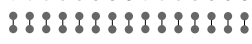
$:: !: !: !: !: !: !: !$

$$
\text { I H-passivated } \mathrm{Si} \text { \& Depassivated Si } \mathrm{PH}_{2}
$$

FIG. 7. Illustrative $\mathrm{PH}_{3}$ dosing outcomes for lithographic templates. 


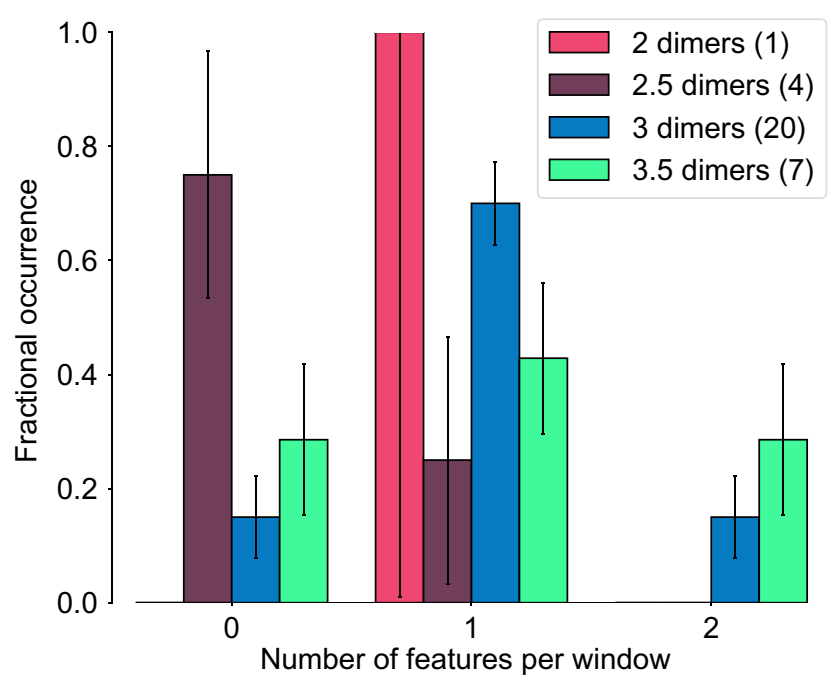

FIG. 8. Donor incorporation statistics expanded to include half dimer windows.

$\operatorname{Si}(100)-2 \times 1$ surface while using empty-state imaging conditions [65].

\section{APPENDIX C: CONSIDERATIONS FOR HALF DIMERS}

While using STM lithography to create three silicon dimer-wide windows $(w=3)$, we often create alternative outcomes such as windows of two $(w=2)$ or four dimer $(w=4)$ width, the inclusion of half dimers, and disordered arrays. We display a selection of common lithographic outcomes in Fig. 6.
After dosing the lithographic patterns with $\mathrm{PH}_{3}$ gas, we examine the adsorption configurations of $\mathrm{PH}_{3}$ fragments in the sites. We observe three general outcomes, as illustrated in Fig. 7: (a) saturation of the lithographic template with $\mathrm{PH}_{3}$ gas fragments, (b) less than saturation coverage of the lithographic template with $\mathrm{PH}_{3}$ gas fragments, and infrequently (c) complete repassivation of the litho site with hydrogen. These observations of sites saturated with $\mathrm{PH}_{3}$ fragments and sites with less than saturation coverage with $\mathrm{PH}_{3}$ fragments occur in spite of dosing conditions, which lead to approximately $0.25 \mathrm{ML}$ P-dopant density for large-area patterns.

In the main text, we compress results for two $(w=2)$ and two and a half dimers $(w=2.5)$ into two dimers, and three $(w=3)$ and three and a half dimer $(w=3.5)$ into three dimers. In Fig. 8, we separate these contributions out. We find that adding a half dimer to the lithographic window tends to decrease the overall rate of incorporation, although the total sample size, particularly for twodimer-wide windows, remains too low to draw significant conclusions.

\section{APPENDIX D: DETAILS OF THE INCORPORATION MODEL}

Our kinetic Monte Carlo model uses reactions and rates that are aggregated from results in the extensive literature on $\mathrm{PH}_{3}$ dissociation on this particular surface $[32,33,40$, 66-68]. In addition to the phosphine configurations and reactions, we add an initial adsorption condition and a concerted reaction.

Our initial condition (which is implicit in Ref. [32], but we are explicitly stating here), relates to (a)

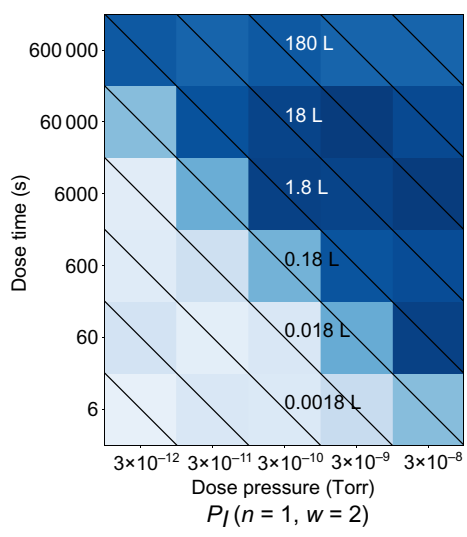

(b)

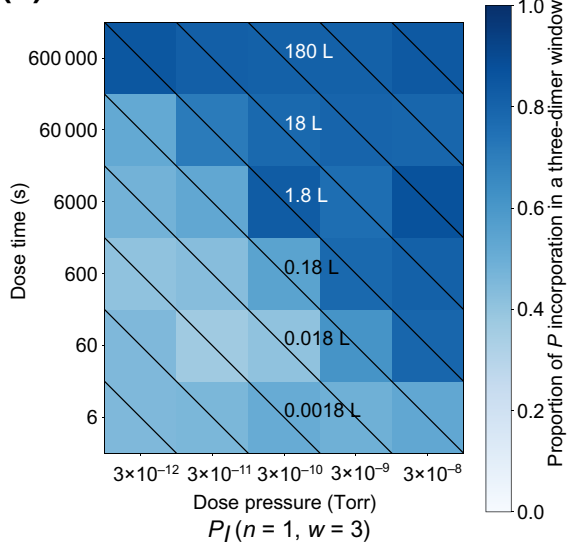

(c)

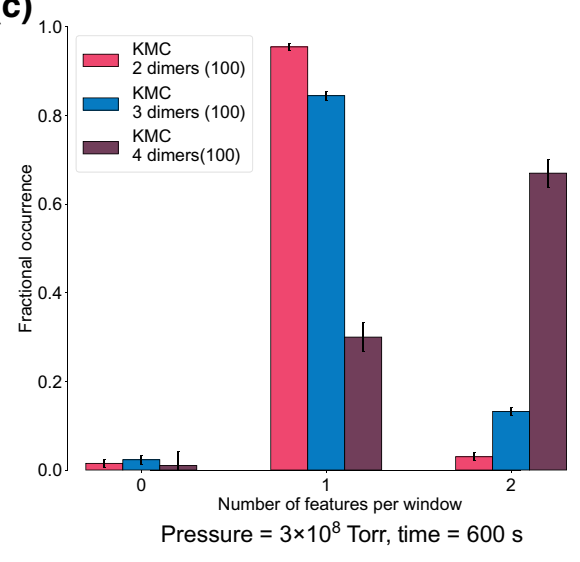

FIG. 9. Incorporation rates for a low-temperature dosing scheme, where the dimer windows are initial dosed at $100 \mathrm{~K}$, and then annealed at $500{ }^{\circ} \mathrm{C}$. (a) The incorporation rate for dimer windows of width $w=2$ as a function of both dosing pressure and temperature. (b) The incorporation rate for dimer windows of width $w=3$ as a function of both dosing pressure and temperature. (c) The relative frequencies of differing numbers of incorporations as a function of dimer width with a dose pressure of $3 \times 10^{-8}$ Torr and a dose time of $600 \mathrm{~s}$. Notably, a significant portion of three-dimer window exposures result in two incorporation events. 


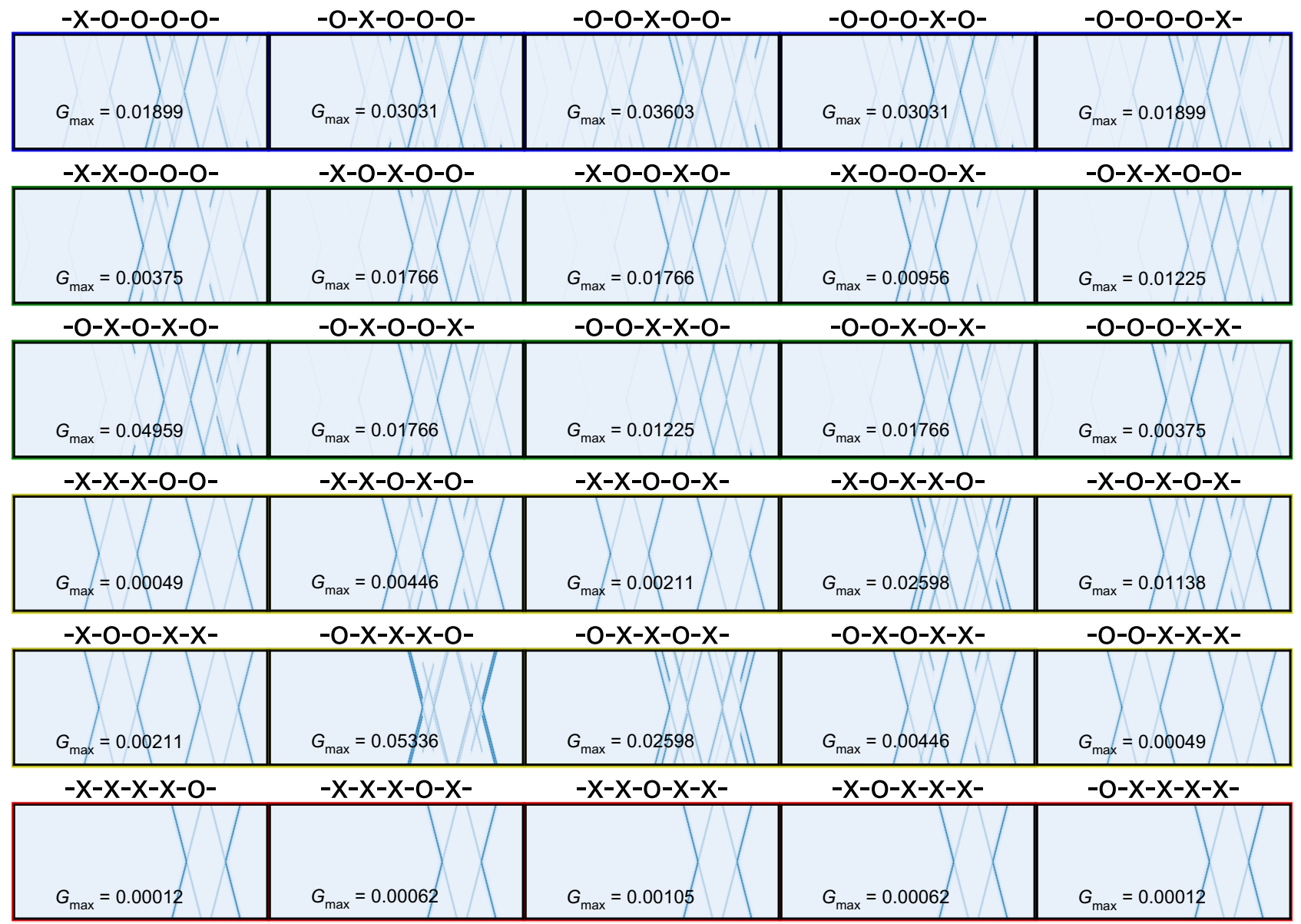

FIG. 10. Exhaustive set of bias spectra for a five-donor chain in which $n \leq 4$ sites are missing. Here the spacing between donors is chosen to be $2.72 \mathrm{~nm}$ along [100]. The $x$ axes are all identical corresponding to varying $\epsilon_{j, \sigma}$ from -0.5 to $0.1 \mathrm{eV}$. The $y$ axes are all identical corresponding to varying the source-drain bias from -0.07 to $0.07 \mathrm{eV}$. The source-chain and drain-chain tunnel rates are fixed at $9.9 \mathrm{meV}$, comparable to the tunnel coupling between two sites, ten lattice spacings apart.

the up-down tilt of silicon dimers a bare $\mathrm{Si}(100)$ $2 \times 1$. We predict phosphine to have an adsorption energy of $-0.91 \mathrm{eV}$ at the lower side of the silicon dimer as opposed to an adsorption energy of $-0.56 \mathrm{eV}$ on the higher side. In our model, we therefore assume that a phosphine molecule will preferentially adsorb on the lower end of the silicon.

We also discover an additional concerted reaction [labeled as B2 to C1 in Fig. 3(b) within the main text]. In this reaction, a $\mathrm{PH}_{2}$ in the favorable lowered dimer position loses a hydrogen atom to a nearby dimer and moves to a bridging $\mathrm{PH}$ position at the same time. This reaction is exothermic with a thermodynamic gain of -1.03 $\mathrm{eV}$ and a barrier of $0.96 \mathrm{eV}$. This contrasts with the similar reaction pathway (B3 to $\mathrm{C} 1$ ) from Warschkow et al. as the $\mathrm{PH}_{2}$ molecule starts in the thermodynamically favored lower end of the silicon dimer (B2) instead of the higher end (B3), which requires an endothermic reaction to move the $\mathrm{PH} 2$ from the favored lowered end (B2) to the higher end (B3) with a thermodynamic cost of $0.24 \mathrm{eV}$ and a barrier of $0.92 \mathrm{eV}$. (While B3 to $\mathrm{C} 1$ is technically a twostep reaction within the Warschkow et al. framework, since the second step is thermodynamically downhill and barrierless, we consolidate it as one reaction within this work.)

Electronic structure theory calculations for these additions are performed with the Gaussian-basis, local-orbital pseudopotential code SEQQUEST.[69] We use a normconserving Perdew-Burke-Ernzerhof (PBE) functional.[70] A seven-layer-thick $4 \times 4$ supercell slab of the $\operatorname{Si}(100)$ $2 \times 1$ is constructed and the dangling bonds at the unreconstructed bottom surface are terminated with selenium atoms, as these are found to minimize strain on the slab. We use a $20 \AA$ vacuum region to ensure the surfaces at either end of the slab are sufficiently isolated from each other. We sample the Brillioun zone with a $2 \times 2 \times 1$ grid. To achieve total energies relaxed within approximately $0.01 \mathrm{eV}$ of the structural minimum, atomic positions are 


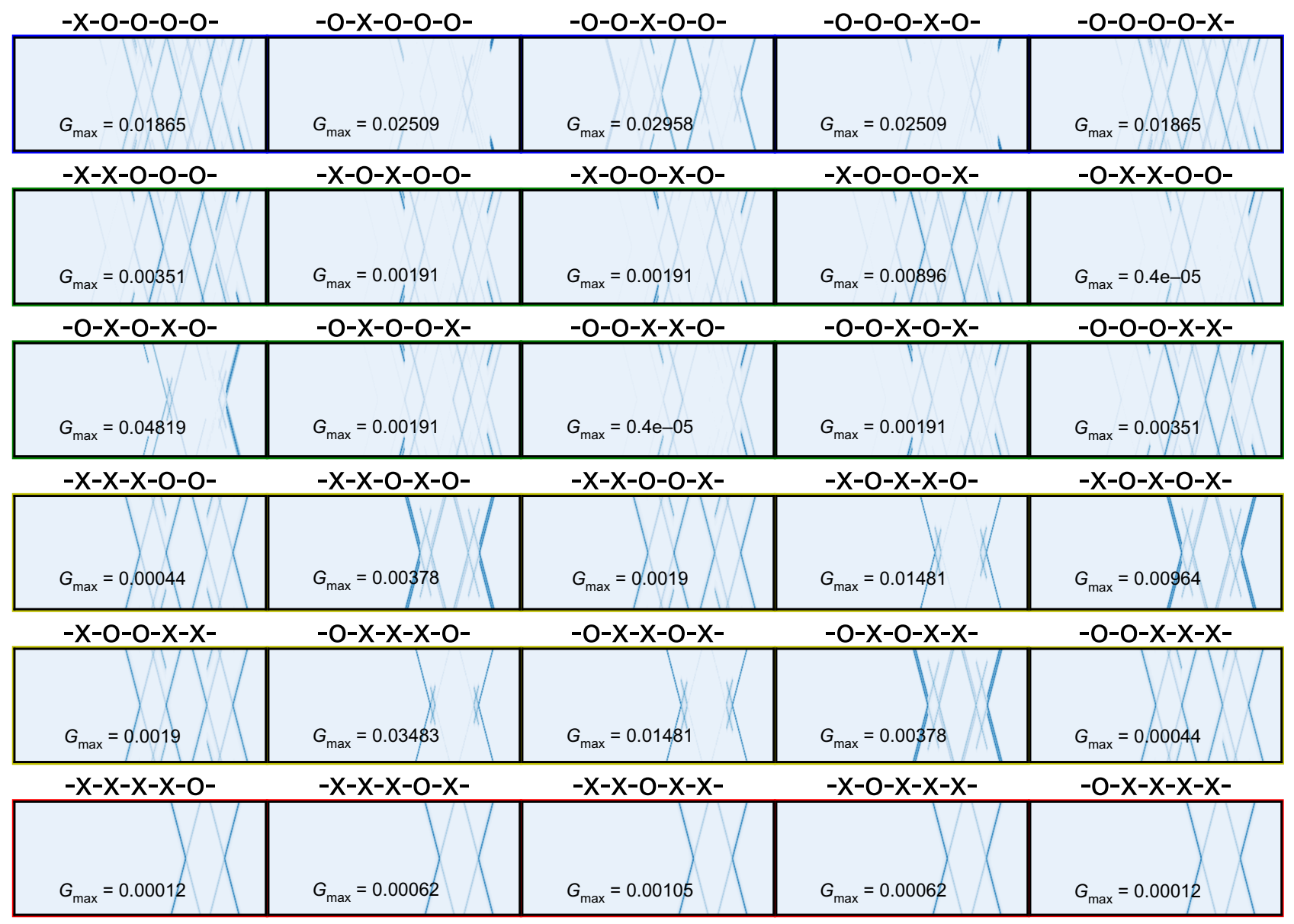

FIG. 11. Exhaustive set of bias spectra for a five-donor chain in which $n \leq 4$ sites are missing. Here the spacing between donors is chosen to be $5.43 \mathrm{~nm}$ along [100]. The $x$ axes are all identical corresponding to varying $\epsilon_{j, \sigma}$ from -0.5 to $0.1 \mathrm{eV}$. The $y$ axes are all identical corresponding to varying the source-drain bias from -0.07 to $0.07 \mathrm{eV}$. The source-chain and drain-chain tunnel rates are fixed at $9.9 \mathrm{meV}$, comparable to the tunnel coupling between two sites, ten lattice spacings apart.

relaxed until the forces are reduced to less than 0.0003 Ry/Bohr.

The barriers described above are then used as inputs in an Arrhenius model [71] for the transition rate $\Gamma=$ $A \exp \Delta / k_{B} T$, where $A$ is the attempt frequency, $\Delta$ is the reaction barrier from DFT, $k_{B}$ is Boltzmann's constant, and $T$ is the temperature. We consider $A=1 \times 10^{12} \mathrm{~s}^{-1}$ for all reactions, which is the order of magnitude calculated for these reactions by Warschkow et al. [32].

We calculate the effusive flow rate of molecules landing on any particular silicon dimer as $\Phi_{\text {effusion }}=$ $\mathrm{PA} / \sqrt{2 \pi m k_{B} T}$, where $P$ is the pressure of the incoming precursor gas, $A$ is the area of impingement, taken here as a single silicon dimer, $m$ is the mass of the precursor gas, $k_{B}$ is the Boltzmann constant, and $T$ is the temperature.

We repeat each KMC calculation 200 times to obtain a meaningful statistical sampling of likely outcomes and report the average outcomes, along with standard deviations as applicable. For ease of reproducibility, we place our kinetic Monte Carlo code on GitHub [72]. We calculate the standard error by assuming a binomial distribution of measured counts and using the standard error based on sample size.

\section{APPENDIX E: LOW-TEMPERATURE DOSING RESULTS}

We also use our kinetic Monte Carlo model to examine low-temperature $(<100 \mathrm{~K})$ dosing techniques to achieve saturation before annealing such as in a recent patent [73]. We simulate a process where the initial dosing step is done at a temperature of $100 \mathrm{~K}$, and subsequent annealing at a temperature of $500^{\circ} \mathrm{C}$ for $15 \mathrm{~s}$. As shown in Fig. 9 in saturated dosing conditions (i.e., $>1$ L coverage), neardeterministic doping can be achieved.

Dosing at low temperatures allows saturation coverage of the exposed window to occur. At $100 \mathrm{~K}$, enough energy is provided for an initially adsorbed phosphine to shed one 


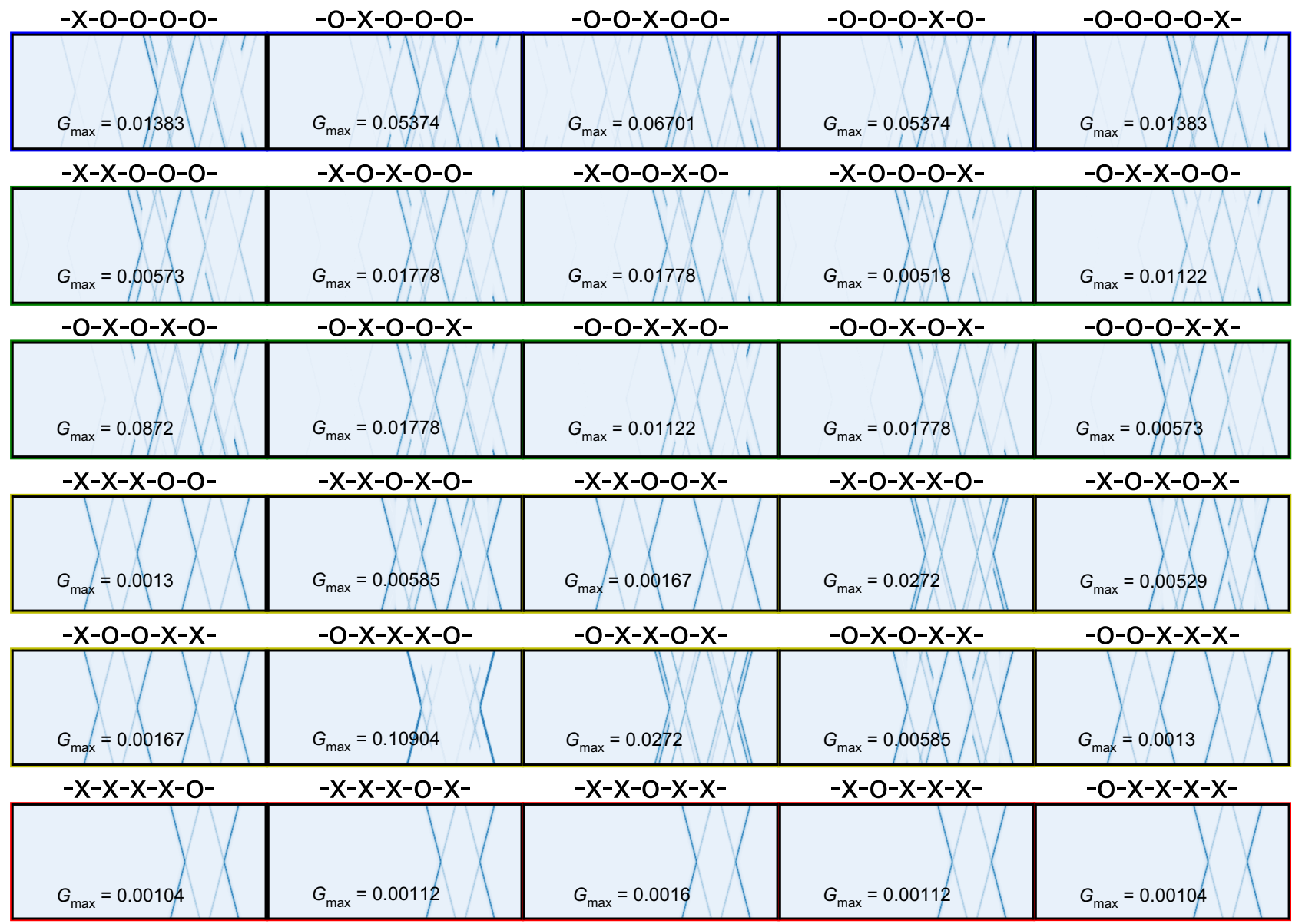

FIG. 12. Exhaustive set of bias spectra for a five-donor chain in which $n \leq 4$ sites are missing. Here the spacing between donors is chosen to be $2.69 \mathrm{~nm}$ along [110]. The $x$ axes are all identical corresponding to varying $\epsilon_{j, \sigma}$ from -0.5 to $0.1 \mathrm{eV}$. The $y$ axes are all identical corresponding to varying the source-drain bias from -0.07 to $0.07 \mathrm{eV}$.

hydrogen, but not for further reactions. The subsequent anneal at $500{ }^{\circ} \mathrm{C}$ provides enough energy for the phosphine fragments to desorb in a reasonable time frame. This provides enough room for the remaining phosphine fragments to fully dissociate into bridging $\mathrm{PH}$ fragments. For a two-dimer-wide window, this process is nearly deterministic with a predicted incorporation rate of $P_{I}(n=1 \mid w=$ 2) $=96 \%$ for a dose pressure of $3 \times 10^{-8}$ Torr and a dose time of $600 \mathrm{~s}$, as shown in Fig. 9(c). However, for the three-dimer window, this process leads to two incorporations with a non-negligible probability; $P_{I}(n=2 \mid w=$ $3)=13 \%$ for a dose pressure of $3 \times 10^{-8}$ Torr and a dose time of $600 \mathrm{~s}$.

While this process can lead to near-deterministic incorporation, it is also highly likely to lead to hydrogen desorption in the surrounding resist. This is due to the barriers for $\mathrm{H}_{2}$ desorption and $\mathrm{PH}_{2}+\mathrm{H}$ desorption being essentially the same. After a low-temperature dose, the entire window will be saturated with $\mathrm{PH}_{2}+\mathrm{H}$ fragments and not full $\mathrm{PH}_{3}$ molecules, making the $\mathrm{PH}_{2}$ desorption barrier the relevant rate limiting step for full dissociation.
Desorption of $\mathrm{PH}_{2}+\mathrm{H}$ has a barrier of approximately $2.0 \mathrm{eV}$, while $\mathrm{H}_{2}$ desorption has a barrier of $2.1 \mathrm{eV}$.[33] Any anneal sufficiently hot to desorb $\mathrm{PH}_{2}$ in a reasonable time frame would therefore almost certainly also desorb hydrogen from the surrounding silicon and destroy the integrity of the surrounding resist. While the small difference in barriers between $\mathrm{PH}_{2}+\mathrm{H}$ and $\mathrm{H}_{2}$ desorption can theoretically be exploited to find an anneal schedule that maximizes $\mathrm{PH}_{2}+\mathrm{H}$ dissociation while leaving $\mathrm{H}_{2}$, we find that this would lead to nondeterministic incorporation. Using a $100-\mathrm{K}$ dose with a $10 \mathrm{~min} 400^{\circ} \mathrm{C}$ anneal had the best effect for maximally encouraging $\mathrm{PH}_{2}+\mathrm{H}$ dissociation with minimal hydrogen-resist degradation, but the maximum incorporation rate is only $P_{I}(n=1 \mid w=3)=$ $79 \%$.

This analysis predicts that saturation dosing at low temperature and then high-temperature annealing techniques are a viable route to deterministic incorporation, albeit with the potentially significant drawback of losing hydrogenresist integrity and thus introducing more uncertainty into the precise location of incorporation. We are careful to note 


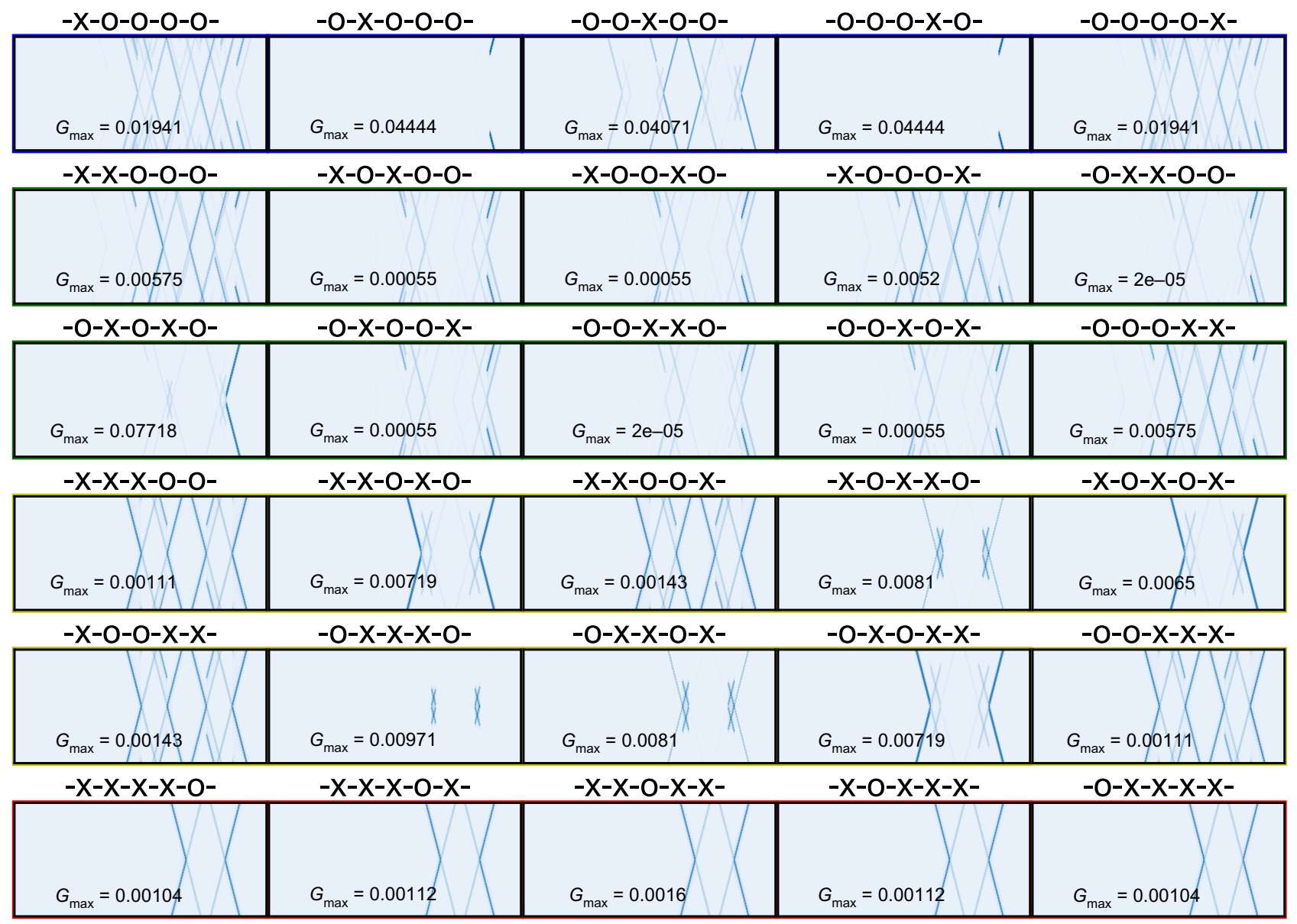

FIG. 13. Exhaustive set of bias spectra for a five-donor chain in which $n \leq 4$ sites are missing. Here the spacing between donors is chosen to be $5.38 \mathrm{~nm}$ along [110]. The $x$ axes are all identical corresponding to varying $\epsilon_{j, \sigma}$ from -0.5 to $0.1 \mathrm{eV}$. The $y$ axes are all identical corresponding to varying the source-drain bias from -0.07 to $0.07 \mathrm{eV}$.

the obvious fact that experiment is the ultimate arbiter of whether the resist integrity is degraded in practice. Combined with the findings in the main text, this indicates that the experimentally stochastic incorporation rates may, in fact, be an artifact of room-temperature dosing. Other regimes of both low- and moderate-temperature dosing with the correct combination of dose pressure and time are predicted to be more feasible routes toward atomic precision deterministic single-donor doping.

\section{APPENDIX F: EXTENDED FERMI-HUBBARD HAMILTONIAN AND TRANSPORT MODELING}

Similar to other authors $[4,5]$, we describe our donor chains with the extended Fermi-Hubbard Hamiltonian, which takes the form

$$
\begin{aligned}
\hat{H}= & \sum_{j, \sigma} \epsilon_{j, \sigma} \hat{n}_{j, \sigma}+\sum_{<j, k>, \sigma} t_{j, k}\left(\hat{c}_{j, \sigma}^{\dagger} \hat{c}_{k, \sigma}+\hat{c}_{k, \sigma}^{\dagger} \hat{c}_{j, \sigma}\right) \\
& +U \sum_{j} \hat{n}_{j, \uparrow} \hat{n}_{j, \downarrow}+\sum_{j, k} V_{j, k} \hat{\rho}_{j} \hat{\rho}_{k} .
\end{aligned}
$$

The four sums comprising this Hamiltonian describe the following physics:

1. The energy $\left(\epsilon_{j, \sigma}\right)$ of a single-particle orbital localized on site $j$ with spin $\sigma$. This includes the effects of externally applied electric fields. The particle-number operator for site $j$ and spin $\sigma$ is $\hat{n}_{j, \sigma}=\hat{c}_{j, \sigma}^{\dagger} \hat{c}_{j, \sigma}$, where $\hat{c}_{j, \sigma}^{\dagger}\left(\hat{c}_{j, \sigma}\right)$ is the associated creation (annihilation) operator associated with that fermionic spin orbital.

2 . The spin-independent tunnel coupling $\left(t_{j, k}\right)$ between sites $j$ and $k$. The brackets in the summation indicate that it is restricted to nearest neighbors, though we note that a more detailed examination of this assumption for arrays with short pitches will be the topic of future work.

3. The Coulomb repulsion $(U)$ associated with double occupation of site $j$ by electrons with opposite spins.

4. The Coulomb repulsion $\left(V_{j, k}\right)$ between electrons occupying sites $j$ and $k$. The density operator associated with site $j, \hat{\rho}_{j}=\hat{n}_{j, \uparrow}+\hat{n}_{j, \downarrow}$. We note that the conventional version of this model is limited to nearest-neighbor intersite repulsion. 
We rely on an implementation of multivalley effective mass theory (MV EMT) [20] developed at Sandia National Laboratories for the parameters of this model. This particular approach to MV EMT due to Shindo and Nara [74] goes beyond the more common Kohn-Luttinger theory [26] and includes a nonperturbative and consistent treatment of both the central-cell correction and valley-orbit coupling that captures the fine structure of the valley-orbit split $1 s$-like states for a single donor. Recent extensions of this implementation of MV EMT that account for electron-electron interactions in the modeling of exchange-coupled donors indicate that this theory can be adjusted to capture the charging energy of a single doubly occupied donor, as well [75]. The $t_{j, k}$ parameters are taken from an exhaustive set of tunnel couplings between individual pairs of donors in bulk silicon that were made available in the arXiv version of Ref. [20,76]. The onsite repulsion is $U=43.8$ $\mathrm{meV}$ to reproduce the charging energy of a single bulklike $D^{-}$state. $V$ is simply the static screened Coulomb interaction between charge centers localized on donor sites with $\epsilon_{\mathrm{Si}}=11.7$.

We expect the accuracy of this particular parameterization will be lacking relative to an experiment for a number of reasons. The tunnel couplings are computed between pairs of bulklike donors, the $U$ term does not account for the fact that the doubly occupied orbitals will have a different character in a chain with a short pitch (i.e., we expect $U$ to be lower), and overall we expect that the singleparticle orbitals will look quite different from our base MV EMT. All of these issues are compounded by the fact that we are studying nonuniform chains in which some sites have failed to incorporate. For the purposes of the qualitative analysis in this paper, we argue that these limitations are acceptable and that experimental design and interpretation will require a more careful parameterization. We note that ongoing work is aimed at developing such a parameterization using a mesh-based MV-EMT capability that makes use of a discontinuous Galerkin discretization of the Shindo-Nara equations and device-specific self-consistent electrostatics. While donor chains are particularly challenging to model with this level of detail, results from this solver (adapted to the Luttinger-Kohn Hamiltonian) have been presented for the analysis of relatively simple Ge-hole quantum dots in Ref. [77].

To simulate bias spectroscopy, we use the MeirWingreen formula [78] to compute the current through a donor chain modeled as an instance of the extended Fermi-Hubbard Hamiltonian in Eq. (F1). We use exact diagonalization to compute the necessary Green's functions, which is computationally tractable for this simplistic model in which each site is comprised of two spin orbitals. A two-point finite-difference formula is used to compute the differential conductance from the current, computed at 501 points for each instance.
We present simulated bias spectra for the exhaustive set of one-dimensional chains with two pitches, corresponding to approximately equal to $2.5-\mathrm{nm}$ and approximately equal to 5-nm spacings along [100] in Figs. 10 and 11 (respectively) and [110] in Figs. 12 and 13. The spacing and length total length of the chain are chosen to be sufficiently short that it is reasonable to expect that the tunneling current can be measured even in the absence of missing sites. We note that our calculations do not include current through scattering states to clearly demarcate the transport through the eigenstates of the chain and thus our Coulomb diamonds do not have signatures of transport "above" or "below" them, as would be expected in a real experiment.

In each of the following figures, the individual rows correspond to a particular number of single-donor sites missing. For a five-donor chain, there are thus 20 distinct nontrivial instances (i.e., five instances with one donor missing, ten with two, ten with three, and five with four). These are indicated on top of each bias spectrum with " $x$ " indicating a missing site and "o" indicating a donor. The scale of the color bars is allowed to vary to capture the relevant details in each plot and only the maximum value of the differential conductance is specified in each panel.

[1] I. M. Georgescu, S. Ashhab, and F. Nori, Quantum simulation, Rev. Mod. Phys. 86, 153 (2014).

[2] E. Prati, M. Hori, F. Guagliardo, G. Ferrari, and T. Shinada, Anderson-mott transition in arrays of a few dopant atoms in a silicon transistor, Nat. Nanotechnol. 7, 443 (2012).

[3] E. Prati, K. Kumagai, M. Hori, and T. Shinada, Band transport across a chain of dopant sites in silicon over micron distances and high temperatures, Sci. Rep. 6, 19704 (2016).

[4] N. H. Le, A. J. Fisher, and E. Ginossar, Extended Hubbard model for mesoscopic transport in donor arrays in silicon, Phys. Rev. B 96, 245406 (2017).

[5] A. Dusko, A. Delgado, A. Saraiva, and B. Koiller, Adequacy of Si: P chains as Fermi-Hubbard simulators, Npj Quantum Inf. 4, 1 (2018).

[6] E. Altman, K. R. Brown, G. Carleo, L. D. Carr, E. Demler, C. Chin, B. DeMarco, S. E. Economou, M. A. Eriksson, and K.-M. C. Fu et al., Quantum simulators: Architectures and opportunities, PRX Quantum 2, 017003 (2021).

[7] H. Büch, S. Mahapatra, R. Rahman, A. Morello, and M. Simmons, Spin readout and addressability of phosphorusdonor clusters in silicon, Nat. Commun. 4, 1 (2013).

[8] C. D. Hill, E. Peretz, S. J. Hile, M. G. House, M. Fuechsle, S. Rogge, M. Y. Simmons, and L. C. Hollenberg, A surface code quantum computer in silicon, Sci. Adv. 1, e1500707 (2015).

[9] P. Pakkiam, M. G. House, M. Koch, and M. Y. Simmons, Characterization of a scalable donor-based singlet-triplet qubit architecture in silicon, Nano Lett. 18, 4081 (2018).

[10] P. Pakkiam, A. Timofeev, M. House, M. Hogg, T. Kobayashi, M. Koch, S. Rogge, and M. Y. Simmons, 
Single-Shot Single-Gate rf Spin Readout in Silicon, Phys. Rev. X 8, 041032 (2018).

[11] Y. He, S. Gorman, D. Keith, L. Kranz, J. Keizer, and M. Simmons, A two-qubit gate between phosphorus donor electrons in silicon, Nature 571, 371 (2019).

[12] M. Koch, J. G. Keizer, P. Pakkiam, D. Keith, M. G. House, E. Peretz, and M. Y. Simmons, Spin read-out in atomic qubits in an all-epitaxial three-dimensional transistor, Nat. Nanotechnol. 14, 137 (2019).

[13] E. Bussmann, R. E. Butera, J. H. Owen, J. N. Randall, S. M. Rinaldi, A. D. Baczewski, and S. Misra, Atomicprecision advanced manufacturing for Si quantum computing, (2021).

[14] T. Škereň, N. Pascher, A. Garnier, P. Reynaud, E. Rolland, A. Thuaire, D. Widmer, X. Jehl, and A. Fuhrer, CMOS platform for atomic-scale device fabrication, Nanotechnology 29, 435302 (2018).

[15] D. R. Ward, S. W. Schmucker, E. M. Anderson, E. Bussmann, L. Tracy, T.-M. Lu, L. N. Maurer, A. Baczewski, D. M. Campbell, M. T. Marshall, and S. Misra, Atomic precision advanced manufacturing for digital electronics, Electron. Device Failure Anal. 22, 4 (2020).

[16] J. Lyding, T.-C. Shen, J. Hubacek, J. Tucker, and G. Abeln, Nanoscale patterning and oxidation of h-passivated Si (100)- $2 \times 1$ surfaces with an ultrahigh vacuum scanning tunneling microscope, Appl. Phys. Lett. 64, 2010 (1994).

[17] S. R. Schofield, N. J. Curson, M. Y. Simmons, F. J. Rueß, T. Hallam, L. Oberbeck, and R. G. Clark, Atomically Precise Placement of Single Dopants in Si, Phys. Rev. Lett. 91, 136104 (2003).

[18] J. Randall, J. Lyding, S. Schmucker, J. Von Ehr, J. Ballard, R. Saini, H. Xu, and Y. Ding, Atomic precision lithography on Si, J. Vac. Sci. Technol. B: Microelectron. Nanometer Struct. Process. Meas. Phenom. 27, 2764 (2009).

[19] L. Oberbeck, N. J. Curson, T. Hallam, M. Y. Simmons, G. Bilger, and R. Clark, Measurement of phosphorus segregation in silicon at the atomic scale using scanning tunneling microscopy, Appl. Phys. Lett. 85, 1359 (2004).

[20] J. K. Gamble, N. T. Jacobson, E. Nielsen, A. D. Baczewski, J. E. Moussa, I. Montaño, and R. P. Muller, Multivalley effective mass theory simulation of donors in silicon, Phys. Rev. B 91, 235318 (2015).

[21] M. Füchsle, Ph.D. thesis, 2011.

[22] A. Ramdas and S. Rodriguez, Spectroscopy of the solidstate analogues of the hydrogen atom: Donors and acceptors in semiconductors, Rep. Prog. Phys. 44, 1297 (1981).

[23] F. Morin, J. Maita, R. Shulman, and N. Hannay, Impurity levels in silicon, Phys. Rev. 96, 833 (1954).

[24] C. Jagannath, Z. Grabowski, and A. Ramdas, Linewidths of the electronic excitation spectra of donors in silicon, Phys. Rev. B 23, 2082 (1981).

[25] A. Mayur, M. D. Sciacca, A. Ramdas, and S. Rodriguez, Redetermination of the valley-orbit (chemical) splitting of the $1 s$ ground state of group-V donors in silicon, Phys. Rev. B 48, 10893 (1993).

[26] W. Kohn and J. Luttinger, Theory of donor states in silicon, Phys. Rev. 98, 915 (1955).
[27] This is a crude boundary that is based on whether the entire state vector of the system could be stored in classical memory. Approximate classical simulation methods can certainly exceed this scale.

[28] For example, we assume that the probability of the sample being misplaced or stolen by a gray jay can be made arbitrarily close to zero.

[29] M. C. Hersam, N. Guisinger, and J. W. Lyding, Isolating, imaging, and electrically characterizing individual organic molecules on the si (100) surface with the scanning tunneling microscope, J. Vac. Sci. Technol. A: Vac. Surf. Films 18, 1349 (2000).

[30] R. Achal, M. Rashidi, J. Croshaw, D. Churchill, M. Taucer, T. Huff, M. Cloutier, J. Pitters, and R. A. Wolkow, Lithography for robust and editable atomic-scale silicon devices and memories, Nat. Commun. 9, 1 (2018).

[31] J. Wyrick, X. Wang, R. V. Kashid, P. Namboodiri, S. W. Schmucker, J. A. Hagmann, K. Liu, M. D. Stewart, C. A. Richter, G. W. Bryant, and R. M. Silver, Atom-by-atom fabrication of single and few dopant quantum devices, Adv. Funct. Mater. 1903475, 1 (2019).

[32] O. Warschkow, N. Curson, S. Schofield, N. Marks, H. Wilson, M. Radny, P. Smith, T. Reusch, D. McKenzie, and M. Y. Simmons, Reaction paths of phosphine dissociation on silicon (001), J. Chem. Phys. 144, 014705 (2016).

[33] H. Wilson, O. Warschkow, N. Marks, N. Curson, S. Schofield, T. Reusch, M. Radny, P. Smith, D. McKenzie, and M. Y. Simmons, Thermal dissociation and desorption of $\mathrm{PH}_{3}$ on $\mathrm{Si}(001)$ : A reinterpretation of spectroscopic data, Phys. Rev. B 74, 195310 (2006).

[34] N. Curson, S. Schofield, M. Simmons, L. Oberbeck, J. O'brien, and R. Clark, STM characterization of the Si-P heterodimer, Phys. Rev. B 69, 195303 (2004).

[35] T. Reusch, N. Curson, S. Schofield, T. Hallam, and M. Simmons, Phosphorus and hydrogen atoms on the (001) surface of silicon: A comparative scanning tunnelling microscopy study of surface species with a single dangling bond, Surf. Sci. 600, 318 (2006).

[36] L. Oberbeck, N. Curson, M. Simmons, R. Brenner, A. Hamilton, S. Schofield, and R. Clark, Encapsulation of phosphorus dopants in silicon for the fabrication of a quantum computer, Appl. Phys. Lett. 81, 3197 (2002).

[37] Y. Wang, M. J. Bronikowski, and R. J. Hamers, Direct dimer-by-dimer identification of clean and monohydride dimers on the Si (001) surface by scanning tunneling microscopy, J. Vac. Sci. Technol. A: Vac. Surf. Films 12, 2051 (1994).

[38] J. Nara, H. Kajiyama, T. Hashizume, Y. Suwa, S. Heike, S. Matsuura, T. Hitosugi, and T. Ohno, Formation Mechanism of One-Dimensional Si Islands on a H/Si(001) Surface, Phys. Rev. Lett. 100, 026102 (2008).

[39] H. Kajiyama, Y. Suwa, S. Heike, M. Fujimori, J. Nara, T. Ohno, S. Matsuura, T. Hitosugi, and T. Hashizume, Roomtemperature adsorption of $\mathrm{Si}$ atoms on H-terminated $\mathrm{Si}$ (001)-2× 1 surface, J. Phys. Soc. Jpn. 74, 389 (2005).

[40] J. M. Bennett, O. Warschkow, N. A. Marks, and D. R. McKenzie, Diffusion pathways of phosphorus atoms on silicon (001), Phys. Rev. B 79, 165311 (2009). 
[41] S. Jeong and A. Oshiyama, Adsorption and Diffusion of Si Adatom on Hydrogenated Si (100) Surfaces, Phys. Rev. Lett. 79, 4425 (1997).

[42] S. Jeong and A. Oshiyama, Complex diffusion mechanisms of a silicon adatom on hydrogenated si (100) surfaces: On terraces and near steps, Surf. Sci. 433, 481 (1999).

[43] This comes from $P(N)=\left[P_{L}(w=2) P_{I}(n=1 \mid w=2)+\right.$ $\left.P_{L}(w=3) P_{I}(n=1 \mid w=3)\right]^{N}=[10 \% \times(40 \% \pm 20 \%)+$ $57 \% \times(63 \% \pm 10 \%)]^{N}=(40 \% \pm 22 \%)^{N}$.

[44] M. Leetmaa and N. V. Skorodumova, KMCLib: A general framework for lattice kinetic monte carlo (KMC) simulations, Comput. Phys. Commun. 185, 2340 (2014).

[45] E. M. Anderson, D. M. Campbell, L. N. Maurer, A. D. Baczewski, M. T. Marshall, T.-M. Lu, P. Lu, L. A. Tracy, S. W. Schmucker, and D. R. Ward et al., Low thermal budget high-k/Metal surface gate for buried donor-based devices, J. Phys.: Mater. 3, 035002 (2020).

[46] These estimates are derived from predicted tunnel couplings between bulklike donors taken from Ref. [20].

[47] J. Van der Heijden, J. Salfi, J. A. Mol, J. Verduijn, G. C. Tettamanzi, A. R. Hamilton, N. Collaert, and S. Rogge, Probing the spin states of a single acceptor atom, Nano. Lett. 14, 1492 (2014).

[48] J. Mol, J. Salfi, R. Rahman, Y. Hsueh, J. Miwa, G. Klimeck, M. Simmons, and S. Rogge, Interface-induced heavyhole/light-hole splitting of acceptors in silicon, Appl. Phys. Lett. 106, 203110 (2015).

[49] R. Ruskov and C. Tahan, On-chip cavity quantum phonodynamics with an acceptor qubit in silicon, Phys. Rev. B 88, 064308 (2013).

[50] T. Kobayashi, J. Salfi, C. Chua, J. van der Heijden, M. G. House, D. Culcer, W. D. Hutchison, B. C. Johnson, J. C. McCallum, and H. Riemann et al., Engineering long spin coherence times of spin-orbit qubits in silicon, Nat. Mater. 20, 1 (2020).

[51] J. Salfi, J. Mol, R. Rahman, G. Klimeck, M. Simmons, L. Hollenberg, and S. Rogge, Quantum simulation of the hubbard model with dopant atoms in silicon, Nat. Commun. 7, 1 (2016).

[52] T. Škereň, S. A. Köster, B. Douhard, C. Fleischmann, and A. Fuhrer, Bipolar device fabrication using a scanning tunnelling microscope, Nat. Electron. 3, 1 (2020).

[53] Q. Campbell, J. A. Ivie, E. Bussmann, S. W. Schmucker, A. D. Baczewski, and S. Misra, A model for atomic precision $p$-type doping with diborane on Si (100)-2 $2 \times 1$, J. Phys. Chem. C 125, 481 (2021).

[54] Q. Campbell, A. D. Baczewski, and S. Misra, Hole in one: Pathways to deterministic atomic-precision single acceptor incorporation on $\mathrm{Si}(100)-2 \times 1$, we hope to have an arXiv number by the time this comes back from review (2021).

[55] K. Dwyer, S. Baek, A. Farzaneh, M. Dreyer, J. Williams, and R. Butera, Area-selective deposition and $\mathrm{B} \delta$-doping of $\mathrm{Si}(100)$ with $\mathrm{BCl}_{3}$, arXiv:2103.07529 (2021).

[56] J. Owen, Q. Campbell, R. Santini, J. Ivie, A. Baczewski, S. Schmucker, E. Bussmann, S. Misra, and J. Randall, Al-alkyls as acceptor dopant precursors for atomic-scale devices, J. Phys.: Condens. Matter 33, 464001 (2021).

[57] M. S. Radue, S. Baek, A. Farzaneh, K. Dwyer, Q. Campbell, A. D. Baczewski, E. Bussmann, G. T. Wang, Y. Mo, and $\mathrm{S}$. Misra et al., $\mathrm{AlCl}_{3}$-Dosed $\mathrm{Si}(100)-2 \times 1$ : Adsorbates,
Chlorinated Al Chains, and Incorporated Al, The Journal of Physical Chemistry C (2021).

[58] M. S. Radue, Y. Mo, and R. Butera, Dopant Precursor Adsorption into Single-Dimer Windows: Towards Guided Self-Assembly of Dopant Arrays on $\mathrm{Si}(100)$, arXiv:2106.10556 (2021).

[59] Y.-L. Hsueh, H. Büch, Y. Tan, Y. Wang, L. C. Hollenberg, G. Klimeck, M. Y. Simmons, and R. Rahman, Spin-Lattice Relaxation Times of Single Donors and Donor Clusters in Silicon, Phys. Rev. Lett. 113, 246406 (2014).

[60] E. Bussmann, R. E. Butera, J. H. Owen, J. N. Randall, S. M. Rinaldi, A. D. Baczewski, and S. Misra, Atomic-precision advanced manufacturing for Si quantum computing, MRS Bull. 46, 1 (2021).

[61] D. R. Ward, M. T. Marshall, D. Campbell, T.-M. Lu, J. C. Koepke, D. A. Scrymgeour, E. Bussmann, and S. Misra, All-optical lithography process for contacting nanometer precision donor devices, Appl. Phys. Lett. 111, 193101 (2017).

[62] E. Bussmann, M. Rudolph, G. Subramania, S. Misra, S. Carr, E. Langlois, J. Dominguez, T. Pluym, M. Lilly, and M. Carroll, Scanning capacitance microscopy registration of buried atomic-precision donor devices, Nanotechnology 26, 085701 (2015).

[63] D.-S. Lin, T.-S. Ku, and R.-P. Chen, Interaction of phosphine with Si (100) from core-level photoemission and real-time scanning tunneling microscopy, Phys. Rev. B 61, 2799 (2000).

[64] S. McKibbin, W. Clarke, A. Fuhrer, T. Reusch, and M. Simmons, Investigating the regrowth surface of Si:P $\delta$-layers toward vertically stacked three dimensional devices, Appl. Phys. Lett. 95, 233111 (2009).

[65] S. Schofield, P. Studer, C. Hirjibehedin, N. Curson, G. Aeppli, and D. Bowler, Quantum engineering at the silicon surface using dangling bonds, Nat. Commun. 4, 1 (2013).

[66] O. Warschkow, H. F. Wilson, N. A. Marks, S. Schofield, N. Curson, P. Smith, M. Radny, D. McKenzie, and M. Y. Simmons, Phosphine adsorption and dissociation on the $\mathrm{Si}(001)$ surface: An ab initio survey of structures, Phys. Rev. B 72, 125328 (2005).

[67] S. R. Schofield, N. J. Curson, O. Warschkow, N. A. Marks, H. F. Wilson, M. Y. Simmons, P. V. Smith, M. W. Radny, D. R. McKenzie, and R. G. Clark, Phosphine dissociation and diffusion on $\mathrm{Si}(001)$ observed at the atomic scale, J. Phys. Chem. B 110, 3173 (2006).

[68] M. Radny, P. Smith, T. Reusch, O. Warschkow, N. Marks, H. Wilson, S. Schofield, N. Curson, D. McKenzie, and M. Y. Simmons, Single hydrogen atoms on the $\mathrm{Si}(001)$ surface, Phys. Rev. B 76, 155302 (2007).

[69] P. A. Schultz, SeqQuest code, see http:/dft.sandia.gov/Quest/.

[70] J. P. Perdew, K. Burke, and M. Ernzerhof, Generalized Gradient Approximation Made Simple, Phys. Rev. Lett. 77, 3865 (1996).

[71] S. Arrhenius, Uber die reaktionsgeschwindigkeit bei der inversion von rohrzucker durch s auren, Zeitschrift für Physikalische Chemie 4, 226 (1889).

[72] Kinetic Monte Carlo code, online at https://github.com/quan tumquinn/apam-kmc. 
[73] M. Simmons and J. Keizer, A method for selective incorporation of dopant atoms in a semiconductive surface (2019).

[74] K. Shindo and H. Nara, The effective mass equation for the multi-valley semiconductors, J. Phys. Soc. Jpn. 40, 1640 (1976).

[75] B. Joecker, A. Baczewski, J. K. Gamble, J. Pla, A. Saraiva, and A. Morello, Full configuration interaction simulations of exchange-coupled donors in silicon using multi-valley effective mass theory, New Journal of Physics (2021).
[76] J. K. Gamble, N. T. Jacobson, E. Nielsen, A. D. Baczewski, J. E. Moussa, I. Montaño, and R. P. Muller, Multivalley effective mass theory simulation of donors in silicon, arXiv:1408.3159 (2014).

[77] A. J. Miller, M. Brickson, W. J. Hardy, C.-Y. Liu, J.-Y. Li, A. Baczewski, M. P. Lilly, T.-M. Lu, and D. R. Luhman, Effective out-of-plane g-factor in strained-Ge/SiGe quantum dots, arXiv:2102.01758 (2021).

[78] Y. Meir and N. S. Wingreen, Landauer Formula for the Current through an Interacting Electron Region, Phys. Rev. Lett. 68, 2512 (1992). 